\title{
THE ROLE OF FLUCTUATIONS IN COARSE-GRAINED DESCRIPTIONS OF NEURONAL NETWORKS*
}

\author{
DAVID CAI ${ }^{\dagger}$, LOUIS TAO ${ }^{\ddagger}$, MAXIM S. SHKARAYEV $§$, AADITYA V. RANGAN $₫$, \\ DAVID W. MCLAUGHLIN", AND GREGOR KOVAČIČ**
}

To Dave Levermore on his 60 th birthday with friendship and appreciation

\begin{abstract}
This paper reviews our recent work addressing the role of both synaptic-input and connectivity-architecture fluctuations in coarse-grained descriptions of integrate-and-fire (I\&F) pointneuron network models. Beginning with the most basic coarse-grained description, the all-to-all coupled, mean-field model, which ignores all fluctuations, we add the effects of the two types of fluctuations one at a time. To study the effects of synaptic-input fluctuations, we derive a kinetictheoretic description, first in the form of a Boltzmann equation in $(2+1)$ dimensions, simplifying that to an advection-diffusion equation, and finally reducing the dimension to a system of two $(1+1)$ dimensional kinetic equations via the maximum entropy principle. In the limit of an infinitely-fast conductance relaxation time, we derive a Fokker-Planck equation which captures the bifurcation between a bistable, hysteretic operating regime of the network when the amount of synaptic-input fluctuations is small, and a stable regime when the amount of fluctuations increases. To study the effects of complex neuronal-network architecture, we incorporate the network connectivity statistics in the mean-field description, and investigate the dependence of these statistics on the statistical properties of the neuronal firing rates for three network examples with increasingly complex connectivity architecture.
\end{abstract}

Key words. Integrate-and-fire neuronal network, kinetic theory, Fokker-Planck equation, meandriven limit.

AMS subject classifications. 82C $31,82 \mathrm{C} 32,92 \mathrm{C} 20,94 \mathrm{C} 15$.

\section{Introduction}

Fluctuations of synaptic inputs, neuronal conductances, and voltages play an important role in the functioning of neuronal networks, from determining individual neurons' spike times and firing rates to possibly being involved in the neural code [6, $29,31,32,65,70,86,97,99,108,117,127,130,139,140]$. Although sufficiently strong stimuli drive a neuronal network into the mean-driven operating regime, in which its dynamics are determined primarily by the mean drive arising from the stimulus and the network response, while the fluctuations in the spike trains arriving at the individual neurons can be neglected, many networks in the brain are believed to

\footnotetext{
*Received: February 14, 2011; accepted (in revised version): August 8, 2011.

${ }^{\dagger}$ Corresponding Author, Department of Mathematics and Institute for Natural Sciences, Shanghai Jiao Tong University, Shanghai 200240, China and Courant Institute of Mathematical Sciences and Center for Neural Science, New York University, 251 Mercer Street, New York, NY 10012-1185, USA (cai@cims.nyu.edu).

${ }^{\ddagger}$ Center for Bioinformatics, National Laboratory of Protein Engineering and Plant Genetics Engineering, College of Life Sciences, Peking University, 5 Summer Palace Road, Beijing 100871, China (taolt@mail.cbi.pku.edu.cn).

${ }^{\S}$ Mathematical Sciences Department, Rensselaer Polytechnic Institute, 110 8th Street, Troy, NY 12180, USA (shkarm@rpi.edu).

ฯ Courant Institute of Mathematical Sciences and Center for Neural Science, New York University, 251 Mercer Street, New York, NY 10012-1185, USA (rangan@cims.nyu.edu).

$\|$ Courant Institute of Mathematical Sciences and Center for Neural Science, New York University, 251 Mercer Street, New York, NY 10012-1185, USA (dmac@cims.nyu.edu).

** Mathematical Sciences Department, Rensselaer Polytechnic Institute, 110 8th Street, Troy, NY 12180, USA (kovacg@rpi.edu).
} 
function in the fluctuation-driven regime, in which synaptic-input fluctuations are the primary cause of neuronal firings $[6,70,86,117,127,139,140]$.

In addition to the input and activity fluctuations, neuronal firing patterns also depend strongly on the underlying network architecture. Far from being uniform, this architecture reflects complex connectivity schemes, which, while always statistical in nature, result in both regular and disordered feature-preference maps $[11,12,16,39,44,60,72,103,116,129,138,142]$. Statistical properties of the network architectural connectivity, such as the probability distributions of the numbers of neurons' pre- and postsynaptic neurons, are likely reflected in the network's functional properties such as neuronal voltage and firing rate distributions, i.e., indicators of functional connectivity [45, 52, 58, 92, 102, 125, 126, 137]. These indicators are typically easier to measure over large scales in vivo, for example using functional magnetic resonance imaging. In particular, such measurements have revealed a possible scale-free distribution, i.e., a distribution with power-law-shaped tails, of neuronal activity correlations between pairs of small volumes in the brain [41,122], hinting at the possibility that individual neuronal connections may follow similar laws.

While large-scale computational modeling that takes into account the dynamics of every individual neuron in a neuronal network has made great strides in the last decade or so $[22,29,33,78,96,97,120,130,131,136]$, it still cannot begin to efficiently describe the dynamics in even one large brain area, such as the entire primary visual cortex. This is because of the large numbers of neurons and vast disparities of both spatial and temporal scales involved in the network processing that takes place in such areas. To be successful in the modeling of various brain area dynamics, one therefore frequently resorts to some degree of coarse-graining. A typical coarse-graining procedure shares much in common with the derivation of the hydrodynamic equations from the Boltzmann kinetic theory of molecular dynamics [14, 15]. It replaces groups of neurons, so small that the neurons contained in them share similar properties and are uniformly connected yet sufficiently large that a statistical description is applicable, by neuronal tissue patches whose dynamics reflects the average neuronal response in the patch $[31,32,95]$. This type of coarse graining also naturally follows from the laminar structure of the brain and the plethora of feature-preference maps $[11,12,16,39,44,60,72,103,116,129,138,142]$.

In addition to computational cost reduction and mathematical simplification, coarse-grained models often furnish a conceptual advantage by providing simple descriptions and explanations of mechanisms underlying the dynamical behavior of more detailed point-neuron models. For example, a simple Fokker-Planck-type coarsegrained model captures a bifurcation between stable, fluctuation-driven, and bistable, mean-driven, network operating regimes, whose understanding has been suggested to be of critical importance in properly tuning a network modeling orientation selectivity in the primary visual cortex $[31,65]$. A yet more basic model has been able to account for neuronal activity patterns in the primary visual cortex during drug-induced visual hallucinations $[17,18]$.

The two main directions in coarse-graining address either the firing rates alone or else also the statistical properties of the relevant neuronal variables such as the membrane potential and conductance. The firing-rate, or mean-field, models $[10,17$, $112,135,144,145]$ are restricted to the mean-driven regime. The statistical models, exemplified by the kinetic theory $[1,4,8,24,31,32,34,36,48,50,55,62,87-91,95,143]$ or the field-theoretic approach [19-21,26-28,93,94], take synaptic-input fluctuations into account, and therefore also provide a description for the network processing of 
weaker stimuli that takes place in the fluctuation-driven regime.

This paper reviews our recent results on the coarse-graining of, and especially our kinetic-theoretic approach to, neuronal network dynamics, addressing both synapticinput and network-connectivity fluctuations [31, 32, 65, 81, 95, 99, 112-114]. We add the effects of these fluctuations one-by-one to the idealized, mean-field network model with all-to-all coupling, which accounts for no fluctuations at all and can be solved exactly in the steady-state, yet provides the backbone structure for all more complicated models [31,32,112]. We then add synaptic-input fluctuations and describe a general method for obtaining a kinetic-theoretic description of integrate-and-fire (I\&F) coupled point-neuron models $[31,32,95,99]$. We focus on all-excitatory networks of simple cells; the treatment for networks also containing inhibitory neurons and complex cells follows along the same lines $[31,95]$. We then discuss how, in the fluctuation-driven regime, the effect of the fluctuations is adequately captured by a simplified Fokker-Planck-type model $[65,81]$. We address adding complexity in the network topology within the framework of the mean-field model, so as to separate its effects from those of synaptic fluctuations [113,114]. For three example networks with complex architecture, we find how the firing-rate statistics reflect the statistics of the underlying network connectivity in the asymptotic limit of strong external driving.

In addressing the kinetic-theoretic approach to neuronal network dynamics, we begin by using a nonequilibrium statistical physics framework to obtain a $(2+1)$ dimensional Boltzmann-type kinetic equation for the probability density function of the neuronal membrane potential and synaptic conductance $[1,8,24,31,32,34,36,48$, $50,55,62,87-91,143]$. We then show how the assumption of statistical independence between the neuronal voltages and conductances, which we can make in the meandriven limit in which all the synaptic-input fluctuations vanish, leads to a mean-field model $[31,112]$. This model is quite accurate in the mean-driven regime [65,81]. We then highlight our important advancement, which was to further reduce the $(2+1)$ dimensional Boltzmann-type kinetic equation to a set of two $(1+1)$-dimensional partial differential kinetic equations in terms of the membrane potential alone [31,32, 95], and thus both reduce computational costs and make the resulting equations more amenable to theoretical analysis. This reduction is achieved via an appropriate closure which follows from the maximum entropy principle [95].

In general, the kinetic equations must be solved numerically. Since the presence of the firing rate in both their coefficients and boundary conditions as a consistency parameter makes the resulting initial-boundary value problem highly nonlinear, it is a non-trivial task to numerically evolve this system. We do not describe the numerical method for solving the kinetic equations here, but instead refer the reader to our work in [98].

A Fokker-Planck equation for the membrane-potential probability density alone follows from the kinetic theory in the limit of very short neuronal conductance timescales [31,65,87], and can also be derived directly from the corresponding singular limit of the I\&F network. We discuss how this equation still takes into account synaptic-input fluctuations, and continuously reduces to a mean-field equation when these fluctuations vanish $[31,65,81]$.

Just like the effects of synaptic-input fluctuations, we describe the effects of complex network-connectivity architecture statistically. We use the graph-theory based description of complex-network topology, which is well-developed and has already found applications ranging from descriptions of the internet to descriptions of social interactions and scientific collaborations $[13,40,42,43,51,82,83,85,128,141]$. The dif- 
ference between the theory of complex neuronal networks and the standard complexnetwork theory is in that, unlike most social and technological networks, neuronal networks must be described by directed graphs, and thus require an additional step in their description. This step consists of deriving the statistics of the directed neuronto-neuron connections from their undirected counterparts [113,114]. For three example networks, we incorporate these statistics into their mean-field description in the high-conductance limit, in order to find the corresponding firing-rate statistics. We solve the resulting linear systems explicitly, and then derive and compare the firing-rate statistics of neurons grouped according to their architectural connectivity properties. In particular, we find that networks with a scale-free distribution of the number of synaptic connections also give rise to a scale-free distribution of the neuronal firing rates. Comparisons with numerical computations involving the intact mean-field model, as well as direct numerical simulations of the corresponding I\&F model, both averaged over many realizations of the network with the prescribed connectivity statistics, confirm our theoretical predictions.

The remainder of the paper is organized as follows. In Section 2.1, we describe the all-to-all coupled, excitatory I\&F neuronal network for which we develop the kinetic theory. In Section 2.2, we present a heuristic derivation of the $(2+1)$ dimensional Boltzmann-type functional differential equation that is the basis of our kinetic-theoretic argument, and derive its boundary conditions. In Section 2.3, we derive the mean-field equations from the Boltzmann equation, solve them explicitly for steady external drive, and discuss the properties of this explicit solution in the meanand fluctuation-driven regimes. In Section 2.4, we simplify the Boltzmann equation into an advection-diffusion equation, and then further reduce this equation to a $(1+1)$-dimensional system of two kinetic equations via the maximal-entropy closure. In Section 2.5 we derive the Fokker-Planck equation in the limit of short conductance times, and discuss how hysteretic behavior of the network firing rate emerges as the synaptic-input fluctuations decrease. In Section 3.1, we derive a generalization of the Boltzmann and mean-field equations to heterogeneously-coupled networks. In Section 3.2, we discuss how in three networks with increasingly complex connectivity topology, the firing rate statistics depend on the network connectivity statistics. In Section 3.3, we extend the mean-field and kinetic theories to spatially-extended networks. Finally, in Section 4, we present a concluding discussion. A flowchart depicting the interdependence of the equations described in this paper is presented in Appendix A.

\section{Synaptic input fluctuations}

We begin our discussion by addressing what role the fluctuations in the synaptic input play in the statistical description of neuronal network dynamics. After deriving, in a heuristic manner, an exact Boltzmann differential-difference equation describing the one-point statistics of the neuronal voltages and conductances in an idealized, all-to-all coupled network, we present a detailed description of the limiting case in which neither synaptic nor architectural-connectivity fluctuations are present in the network. The mean-field equation describing this limiting case is solved exactly and gives a complete statistical description of the idealized, fluctuationless network. We then proceed to derive a kinetic-theoretic description of the network that takes synaptic-input fluctuations into account. After simplifying the Boltzmann differentialdifference equation into an advection-diffusion equation using the diffusion approximation, we derive a kinetic-theoretic description of the neuronal voltage statistics alone via the maximum-entropy principle. This description consists of two equations for the 
voltage probability density function (pdf) and the first moment of the conductance. We show how the standard Fokker-Planck equation used to describe neuronal voltage statistics when the conductance time-scale vanishes follows from the kinetic-theoretic description. We finally discuss how the fluctuationless, mean-field description can be derived as a limiting case of all of the above descriptions.

2.1. All-to-all coupled excitatory $\mathbf{I} \& \mathbf{F}$ network. To first exclude any effects of network-connectivity fluctuations, we begin by studying the dynamics of an all-to-all coupled network. For clarity of presentation, and since the extension of our techniques to networks containing inhibitory neurons is straightforward, we only consider an excitatory network. The dynamics of such an all-to-all coupled, excitatory, I\&F neuronal network, consisting of $N$ neurons is governed by the set of equations

$$
\begin{aligned}
\tau \frac{d V_{i}}{d t} & =-\left(V_{i}-\varepsilon_{r}\right)-G_{i}\left(V_{i}-\varepsilon_{E}\right), \\
\sigma \frac{d G_{i}}{d t} & =-G_{i}+f \sum_{\mu} \delta\left(t-t_{\mu}^{i}\right)+\frac{S}{N} \sum_{j \neq i} \sum_{\mu} p_{i j \mu} \delta\left(t-t_{j \mu}\right),
\end{aligned}
$$

for $i=1, \ldots, N$, where $V_{i}(t)$ is the membrane potential of the $i$ th neuron in the network, $G_{i}(t)$ is its excitatory conductance, $\tau$ is the time scale of the membrane potential and $\sigma$ of the excitatory conductance, $\delta(\cdot)$ is the Dirac delta function, $f$ is the strength of the external-drive spikes, $S$ is the strength of coupling between neurons in the network, $\varepsilon_{E}$ the excitatory reversal potential, and $\varepsilon_{r}<\varepsilon_{E}$ is the leakage reversal potential. For simplicity, $\varepsilon_{r}$ is also taken to equal the reset potential, but the kinetic-theoretic approach presented here can also be carried out when these two potentials are different. The variable $p_{i j \mu}$ describes the probability of synaptic release, which is modeled by a Bernoulli process with release probability $p$. In other words, $p_{i j \mu}=1$ with probability $p$, and 0 with probability $1-p$.

As alluded to in the previous paragraph, the two delta-function trains in Equation (2.1b) represent the spikes arriving from the external drive and other neurons in the network, respectively. We assume that the $i$ th neuron receives spikes from the external input at times $\left\{t_{\mu}^{i}\right\}$, which form an independent realization of the Poisson process with rate $\nu(t)$. Each incoming external spike induces a jump of size $f / \sigma$ in the conductance of the receiving neuron. At the spike-time $t_{i \mu}$, when the $i$ th neurons's voltage $V_{i}(t)$ crosses the firing threshold $V_{T}$, the voltage $V_{i}(t)$ is instantaneously reset to the reset voltage $\varepsilon_{r}$, and spikes are sent to all other neurons in the network. These spikes induce jumps of size $S / N \sigma$ in the conductances of each of their postsynaptic neurons. Note that physiology implies the inequality $\varepsilon_{r}<V_{T}<\varepsilon_{E}$. We assume that the generation and transmission of the network spikes is instantaneous, i.e., there is no refractory period. The factor $1 / N$ ensures a finite network firing rate $N m(t)$ in the limit of $N \rightarrow \infty$. Here, $m(t)$ is the population-averaged firing rate per neuron in the network.

While the output spike-train of a fixed neuron in general does not obey Poisson statistics, the spike train obtained from the summed output of all neurons in the network does obey these statistics asymptotically for large network size $N$, provided we assume that each neuron fires at a very low rate and its spike times are statistically independent from one-another [37]. We will therefore assume that the summed input spike train from all other neurons to the $i$ th neuron is Poisson with rate $p N m(t)$, where $p N$ is the effective number of neurons coupled to the $i$ th neuron, in other words, the effective network size in the all-to-all coupled network. 
We remark that we consider the all-to-all network connectivity here only for the ease of discussion; the derivation can be generalized to to more general types of network connectivity, as we will see in Section 3.

2.2. The Boltzmann equation. To study the one-point statistical behavior of the network (2.1), we construct a statistical ensemble of its identical copies differing only in their initial voltages $V_{i}(0)$ and conductances $G_{i}(0), i=1, \ldots, N$, and their inputs. Each input is an independent set of $N$ independent realizations of the Poisson spike train with the same rate $\nu(t)$, with each realization feeding a different neuron. We are interested in the probability of finding a neuron whose voltage lies in the interval $(v, v+d v)$ and whose conductance lies in the interval $(g, g+d g)$ at time $t$, described in terms of the corresponding probability density as $\rho(v, g, t) d v d g$.

Here, we present a heuristic derivation of the equation governing the evolution of the probability density $\rho(v, g, t)$. At time $t$, in the $(v, g)$-phase space, the probability of a given neuron's voltage and conductance to reside in the infinitesimal rectangle whose sides are the intervals $(v, v+d v)$ and $(g, g+d g)$, respectively, is $\rho(v, g, t) d v d g$. During the time interval $(t, t+d t)$, this probability changes due to

(i) the streaming of the phase points through the boundaries of this rectangle under the smooth dynamics of Equation (2.1) in the absence of any spikes;

(ii) the conductance jumps induced by the external and network spikes, described by the trains of Dirac delta functions in Equation (2.1b).

As seen from Equation (2.1), the smooth streaming in the $v$-direction through the sides of the rectangle at $v$ and $v+d v$ contributes the change

$$
\begin{aligned}
& -\left[\left(\frac{v-\varepsilon_{r}}{\tau}\right)+g\left(\frac{v-\varepsilon_{E}}{\tau}\right)\right] \rho(v, g, t) d g d t \\
& \quad+\left[\left(\frac{v+d v-\varepsilon_{r}}{\tau}\right)+g\left(\frac{v+d v-\varepsilon_{E}}{\tau}\right)\right] \rho(v+d v, g, t) d g d t .
\end{aligned}
$$

Note that we have neglected the variations of the density $\rho$ in the conductance $g$ over two sides at $v$ and $v+d v$, since their contributions to the probability change are of higher order. Likewise, the smooth streaming in the $g$-direction through the sides of the rectangle at $g$ and $g+d g$ contributes the change

$$
-\frac{g}{\sigma} \rho(v, g, t) d v d t+\frac{g+d g}{\sigma} \rho(v, g+d g, t) d v d t
$$

where, again, we have neglected the variations in $v$ over these two sides.

The jumps due to the external spikes contribute the abrupt change

$$
\left[\rho\left(v, g-\frac{f}{\sigma}, t\right)-\rho(v, g, t)\right] d v d g d t
$$

at the time-rate $\nu(t)$. Likewise, the jumps due to the network spikes contribute the change

$$
\left[\rho\left(v, g-\frac{\bar{S}}{p N \sigma}, t\right)-\rho(v, g, t)\right] d v d g d t
$$

at the time-rate equal to the network firing-rate, $p N m(t)$. In this formula, $\bar{S}=p S$. It is here that we need the assumption that the spike trains arriving at any given 
neuron obey the Poisson statistics. In particular, the probability of a spike arrival causing a jump in the density $\rho(v, g, t)$ over the time $d t$ is $\nu(t) d t$ and $p N m(t) d t$ for external-drive and network spikes, respectively.

The overall change of $\rho(v, g, t)$ during the time interval $(t, t+d t), d \rho(v, g, t) d v d g$, equals the sum of all the terms in the expressions (2.2), with the last two terms multiplied by the appropriate time-rates. Dividing the equation obtained in this way by $d v d g d t$, and letting $d v \rightarrow 0, d g \rightarrow 0$ and $d t \rightarrow 0$, we thus arrive at the following Boltzmann equation governing the evolution of the probability density $\rho=\rho(v, g, t)$ :

$$
\begin{aligned}
\partial_{t} \rho=\partial_{v} & \left\{\left[\left(\frac{v-\varepsilon_{r}}{\tau}\right)+g\left(\frac{v-\varepsilon_{E}}{\tau}\right)\right] \rho\right\}+\partial_{g}\left(\frac{g}{\sigma} \rho\right) \\
& +\nu(t)\left[\rho\left(v, g-\frac{f}{\sigma}, t\right)-\rho(v, g, t)\right] \\
& +p N m(t)\left[\rho\left(v, g-\frac{\bar{S}}{p N \sigma}, t\right)-\rho(v, g, t)\right],
\end{aligned}
$$

valid for $\varepsilon_{r}<v<V_{T}$ and $0<g<\infty$. From the above discussion it follows that the first two terms (2.3a) in Equation (2.3) describe the streaming dynamics of the neurons without receiving any spikes, while the third and fourth terms, (2.3b) and (2.3c), describe the conductance jumps of the neurons at the times they have received external input spikes and spikes from other neurons in the network, respectively.

Note that the derivation of Equation (2.3) was carried out entirely in the $(v, g)$ phase-space of a single neuron, and the influence of other neurons was only taken into account through the factor $p N m(t)$ in the term (2.3c). This should be clear as we assume all the neurons to be statistically equivalent, and their only interaction to take place through spikes. Note also that the Boltzmann Equation (2.3) is not exact, due to the summed input from all the neurons in the network being only approximately Poisson, and thus the term $(2.3 \mathrm{c})$ is valid only in an asymptotic sense. A more mathematically rigorous derivation of the Boltzmann Equation (2.3) is given in the appendix of [31].

The Boltzmann Equation (2.3) can be written in the conservation-of-probability from,

$$
\partial_{t} \rho+\partial_{v} J_{V}(g, v)+\partial_{g} J_{G}(g, v)=0,
$$

with the corresponding membrane-potential and conductance probability fluxes

$$
\begin{aligned}
& J_{V}(v, g)=- {\left[\left(\frac{v-\varepsilon_{r}}{\tau}\right)+g\left(\frac{v-\varepsilon_{E}}{\tau}\right)\right] \rho(v, g), } \\
& J_{G}(v, g)=-\frac{g}{\sigma} \rho(v, g)+\nu(t) \int_{g-f / \sigma}^{g} \rho(v, h) d h \\
&+p N m(t) \int_{g-\bar{S} / p N \sigma}^{g} \rho(v, h) d h,
\end{aligned}
$$

respectively.

To derive the boundary conditions for Equation (2.3), we recall from Section 2.1 that once a neuron's voltage crosses the threshold $V_{T}$ it is instantaneously reset to $\varepsilon_{r}$, while the value of the conductance remains unchanged. Mathematically, this fact corresponds to the condition

$$
J_{V}\left(V_{T}, g\right)=J_{V}\left(\varepsilon_{r}, g\right)
$$


for $0 \leq g<\infty$. In addition, since no neuronal conductance ever becomes negative, we must have

$$
\rho(v, g<0) \equiv 0
$$

Likewise, since no neuronal conductance ever becomes infinite, the density $\rho(v, g)$ must vanish at large infinite conductance values,

$$
\rho(v, g \rightarrow \infty) \rightarrow 0
$$

sufficiently rapidly, together with all its derivatives, for $\varepsilon_{r} \leq v<V_{T}$. Equations (2.6a), $(2.6 \mathrm{~b})$, and $(2.6 \mathrm{c})$ constitute the boundary conditions for the Boltzmann Equation $(2.3)$

To obtain a self-consistent kinetic description of the dynamics exhibited by the I\&F system (2.1), we finally need to determine the evolution of the populationaveraged firing rate, $m(t)$. This rate is given by the total probability flux across the threshold regardless of the conductance values, i.e.,

$$
\begin{aligned}
m(t) & =\int_{0}^{\infty} J_{V}\left(V_{T}, g, t\right) d g \\
& =-\int_{0}^{\infty}\left[\left(\frac{V_{T}-\varepsilon_{r}}{\tau}\right)+g\left(\frac{V_{T}-\varepsilon_{E}}{\tau}\right)\right] \rho\left(V_{T}, g, t\right) d g .
\end{aligned}
$$

Once the solution for the probability density $\rho(v, g, t)$ is known, we can determine the firing rate $m(t)$ via Equation (2.6d). On the other hand, Equation (2.3) contains the firing rate $m(t)$ as a multiplicative parameter. Since the firing rate $m(t)$, in turn, depends on the boundary value of $\rho\left(V_{T}, g, t\right)$ through Equation $(2.6 \mathrm{~d})$, the boundaryvalue problem specified by Equation (2.3) and the boundary conditions (2.6) is clearly nonlinear.

2.3. The mean-driven limit. The simplest, most highly idealized model of neuronal network dynamics ignores both its network-architecture and synaptic-input fluctuations. In all-to-all connected networks, which we have been studying so far, we have already excluded all the network-architecture fluctuations. To also exclude the synaptic-input fluctuations, we now study the limit in which the mean of the synaptic input drives the network neurons to fire, while the effect of the synapticinput fluctuations is negligible. This limit is referred to as the mean-driven limit. Intuitively, one would expect the network to operate in this limit when each neuron receives a large number of spikes from both the external and network drive, each of which only induces a very small conductance change. If we restrict our interest to bounded neuronal firing rates, this limit also implies large network size. Consequently, in this limit, we must let

$$
f \rightarrow 0, \quad N \rightarrow \infty, \quad \nu(t) \rightarrow \infty, \quad f \nu(t)=\mathcal{O}(1)
$$

with the last condition imposed so that the mean synaptic input remains finite.

2.3.1. Closure in the mean-driven limit. As seen directly from the network (2.1) and conditions (2.7), in the mean-driven limit, the conductance is the same for all neurons, i.e., the effect of the second term $f \sum_{\mu} \delta\left(t-t_{\mu}^{i}\right)$ in Equation (2.1b) is equivalent to a smooth input $f \nu(t)$, whereas the third term, which signifies the network interaction in Equation (2.1b), is equivalent to a smooth input $\bar{S} m(t)$ to a 
neuron from all other neurons in the network, with the corresponding fluctuations scaled away at the rate $1 / \sqrt{N}$.

Under these smooth, fluctuation-free conductance inputs, if the external drive is sufficiently strong, Equations (2.1) imply that the neuronal voltages quickly increase from the reset $\varepsilon_{r}$ to the threshold $V_{T}$ after which the neurons fire. This observation suggests that, statistically, the value of a neuron's conductance provides little information about the value of its voltage in this limit. The reason is that, in this limiting regime and under strong drive, conductance values are typically high, and so relatively small conductance fluctuations exert little influence on voltage dynamics. One should thus expect in the mean-driven limit that the dynamics of conductance and voltage are uncorrelated. Consequently, we can reasonably assume the dynamics of the neuronal conductances and voltages to be statistically independent,

$$
\rho(v, g, t)=\rho^{(v)}(v, t) \rho^{(g)}(g, t) .
$$

Denoting the average conductance by

$$
\langle g\rangle(t) \equiv \int_{0}^{\infty} g \rho^{(g)}(g, t) d g
$$

and integrating the Boltzmann Equation (2.3) over the conductance $g$, together with the boundary condition (2.6c), we find the equation

$$
\partial_{t} \rho^{(v)}(v)=\partial_{v}\left\{\left[\left(\frac{v-\varepsilon_{r}}{\tau}\right)+\langle g\rangle\left(\frac{v-\varepsilon_{E}}{\tau}\right)\right] \rho^{(v)}(v)\right\} .
$$

Likewise, multiplying Equation (2.3) by the conductance $g$ and then integrating it over both the voltage and conductance, together with the flux boundary condition (2.6a), we find the equation

$$
\frac{d}{d t}\langle g\rangle=-\frac{1}{\sigma}[\langle g\rangle-\bar{g}(t)]
$$

where

$$
\bar{g}(t)=f \nu(t)+\bar{S} m(t)
$$

is the mean synaptic input to a neuron. Note that Equation (2.10a) can be written in the conservation form $\partial_{t} \rho^{(v)}(v, t)+\partial_{v} j_{V}(v, t)=0$, with the probability flux

$$
j_{V}(v)=-\left[\left(\frac{v-\varepsilon_{r}}{\tau}\right)+\langle g\rangle\left(\frac{v-\varepsilon_{E}}{\tau}\right)\right] \rho^{(v)}(v) .
$$

Equations (2.10) are closed with respect to $\rho^{(v)}(v, t)$ and $\langle g\rangle(t)$ : Equation (2.10a) is a $(1+1)$-dimensional PDE that describes the evolution of the voltage pdf $\rho^{(v)}(v, t)$, whereas Equation (2.10b) describes the evolution of the average conductance $\langle g\rangle(t)$. The boundary conditions (2.6a) and (2.6d) for the Boltzmann equation now yield the sole boundary condition

$$
j_{V}\left(\varepsilon_{r}\right)=j_{V}\left(V_{T}\right)=m(t)
$$

needed for the system (2.10). 
2.3.2. Explicit solution in the stationary case. In the time-homogeneous case of the mean-driven limit, i.e., the case in which the Poisson rate $\nu$ of the external input does not vary with time, the system (2.10) simplifies to the point that the resulting problem can be solved exactly. Specifically, since the steady solution of Equation (2.10b) is clearly $\langle g\rangle=\bar{g}$, an integration of the time-independent version of Equation (2.10a), together with Equation (2.13), yields the equation

$$
\rho^{(v)}(v)=-\frac{\tau m}{\left(v-\varepsilon_{r}\right)+\left(v-\varepsilon_{E}\right) \bar{g}},
$$

which holds for $\varepsilon_{r}<v<V_{T}$.

For superthreshold values of the mean synaptic input, $\bar{g}>\bar{g}_{0}$, with

$$
\bar{g}_{0}=\frac{V_{T}-\varepsilon_{r}}{\varepsilon_{E}-V_{T}}
$$

from the normalization condition

$$
\int_{\varepsilon_{r}}^{V_{T}} \rho^{(v)}(v) d v=1
$$

and the fact that the denominator in Equation (2.14) must be negative to ensure $\rho^{(v)}(v) \geq 0$, we find the equation

$$
m=\frac{1+\bar{g}}{\tau \log \left|\frac{\bar{g}\left(\varepsilon_{r}-\varepsilon_{E}\right)}{\left(V_{T}-\varepsilon_{r}\right)+\bar{g}\left(V_{T}-\varepsilon_{E}\right)}\right|} .
$$

Although an explicit input-output relation $m(f \nu)$ between the firing rate $m$ and the external-drive strength $f \nu$ cannot be calculated from Equation (2.17a), an exact parametrization of the gain curve representing it can be obtained in terms of the parameter $\bar{g}$. This parametrization consists of Equation (2.17a) and the equation

$$
f \nu=\bar{g}-\frac{\bar{S}(1+\bar{g})}{\tau \log \left|\frac{\bar{g}\left(\varepsilon_{r}-\varepsilon_{E}\right)}{\left(V_{T}-\varepsilon_{r}\right)+\bar{g}\left(V_{T}-\varepsilon_{E}\right)}\right|},
$$

which follows immediately from the definition (2.11) [65]. In particular, for every $\bar{g}>\bar{g}_{0}$, Equations (2.17) give the value of the firing rate $m$ corresponding to the appropriate value of the input $f \nu$.

2.3.3. Mean- and fluctuation-driven regimes. In the stationary case with no synaptic-input fluctuations, the $m-f \nu$ gain curve must consist of two parts, corresponding to two network operating regimes, as shown in Figure 2.1. First, in the case $\bar{g}<\bar{g}_{0}$, with $\bar{g}_{0}$ as in Equation (2.15), we derive directly from the system (2.1) that all the neuronal membrane potentials approach the equilibrium value

$$
v=V_{S}=\frac{\varepsilon_{r}+\bar{g} \varepsilon_{E}}{1+\bar{g}},
$$

which is known as the effective reversal potential. Therefore, for large times $t$,

$$
\rho^{(v)}(v) \rightarrow \delta\left(v-V_{S}\right) .
$$


The inequality $\bar{g}<\bar{g}_{0}$ immediately implies that $V_{S}<V_{T}$ in this regime, so that all the neuronal voltages are concentrated at one subthreshold value and no neuron fires. Thus the firing rate $m$ vanishes.

The first part of the gain curve therefore consists of the straight-line segment $m=0$ stretching between the external-drive values $f \nu=0$ and $f \nu=\bar{g}_{0}$. This part corresponds to the fluctuation-driven regime, in which the neuronal firings are entirely driven by the voltage fluctuations that result from the synaptic-input fluctuations. As expected, since it operates in the limit of vanishing fluctuations, the network ceases to fire in this regime altogether.

In the mean-driven regime, $\bar{g}>\bar{g}_{0}$, the discussion in the previous section reflects the fact that the network fires at a nonvanishing rate, expressed through the parametrization (2.17), and represented by the second, nontrivial, part of the gain curve. Unless the network is uncoupled, $\bar{S}=0$, the graph of $m$ versus $f \nu$ begins at the point $(f \nu, m)=\left(\bar{g}_{0}, 0\right)$, proceeding backwards in $f \nu$, with the initial derivative $-1 / \bar{S}$. For large values of $\bar{g}$ (and so large external-drive strength $f \nu$, firing rate $m$, or both), every gain curve asymptotes towards its own straight line

$$
m=\frac{f \nu+1-\bar{g}_{0} / \ln \left(1+\bar{g}_{0}\right)}{\tau \ln \left(1+\bar{g}_{0}\right)-\bar{S}} .
$$

For sufficiently weak coupling, $\bar{S}<\tau \ln \left(1+\bar{g}_{0}\right)$, the gain curve turns around in a saddlenode bifurcation, and thus passes through a bistability interval that begins at the saddle-node bifurcation point and ends at the driving threshold $f \nu=\bar{g}_{0}$. For strong coupling, $\bar{S}>\tau \ln \left(1+\bar{g}_{0}\right)$, the gain curve has a negative slope everywhere and terminates with a nonzero firing rate $m$ at $f \nu=0$. In this latter case, the coupling is too strong for the network (2.1) to reach a stable stationary operating state. This scenario is illustrated in the left panel in Figure 2.1.
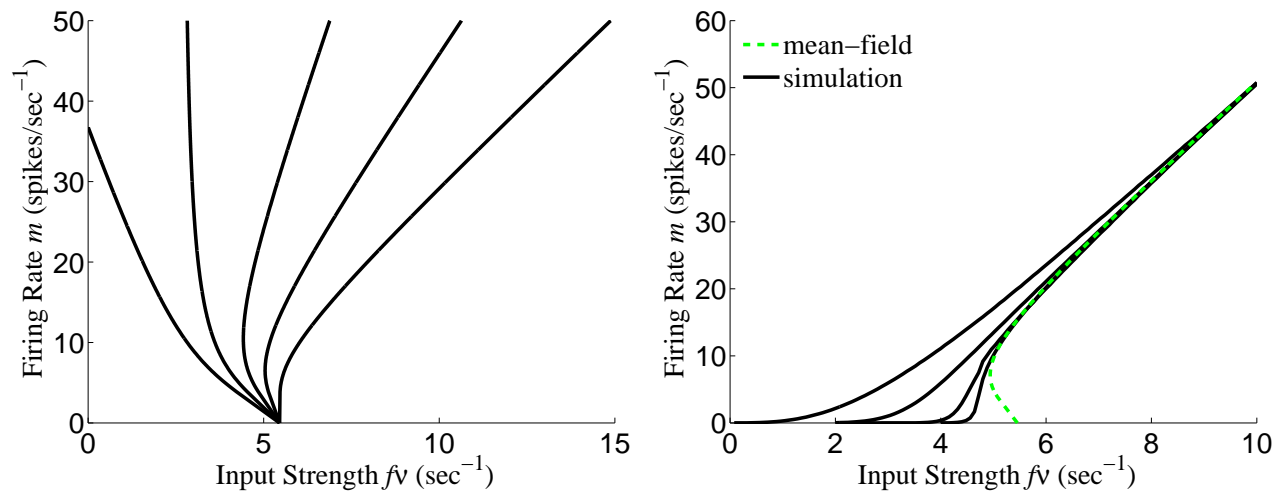

FIG. 2.1. Left: Gain curves of the mean-field model, plotted using the exact parametrization (2.17). The coupling constant $S$ increases from the right to the left curve, starting with $S=0$ along the rightmost curve. The second curve from the left has a vertical asymptote. Right: Gain curves of the IEF system (2.1) compared to the corresponding mean-field gain curve (2.17). The external spike strength $f$ decreases and network size $N$ increases from the left to the right curve, effectively decreasing the amount of synaptic-input fluctuations. [Reproduced with permission from Ref. [65], (Copyright 2009, by APS).]

As can be seen from the right panel in Figure 2.1, the numerically-computed gain curves of the full I\&F system (2.1) approach the mean-field gain curve (2.17) 
at sufficient values of the external drive $f \nu$ in the limit of small conductance-jump sizes and large network size. More surprisingly, in the limit of large external drive $f \nu \rightarrow \infty$, the true gain curves converge onto the mean-field gain curve regardless of the conductance-jump size. On the other hand, the mean-driven limit is evidently insufficient to yield detailed information about the network firing rate in the fluctuationdriven regime. The full kinetic equations, as derived below, or at least a different further approximation, must be used to obtain this information instead. One such further approximation, discussed in Section 2.5, is given by a Fokker-Planck equation. Finally, as we will see in what follows, bistability is only present for small values of synaptic-input fluctuations, and disappears as they increase $[65,81,130]$.

We should remark that in both regimes, the steady conductance distribution is centered sharply around its mean value,

$$
\rho^{(g)}(g)=\delta(g-\bar{g})
$$

which is in accordance with the absence of conductance fluctuations in the steady case of the mean-driven limit.

2.4. Kinetic theory. In the regime of moderate and especially low synaptic input, the mean-field model is obviously inadequate to capture the details of the neuronal gain curves even in the case of very small synaptic-input fluctuations, as evident from the right panel in Figure 2.1. Clearly, as the amount of these fluctuations increases, the assumption (2.8) of statistical independence between the neuronal voltages and conductances grows progressively less accurate, and a different approximate description must be found. We review one such description, furnished by the kinetic theory, in this section.

2.4.1. Diffusion Approximation. To simplify the analysis of the $(2+1)$ dimensional partial-differential/functional Boltzmann Equation (2.3) and uncover the meaning of its solutions for the dynamics of the network (2.1), we now invoke a smalljump approximation and simplify Equation (2.3) to become a $(2+1)$-dimensional, nonlinear advection-diffusion equation. Further on, in Section 2.4.4, we will describe a reduction of the dynamics in two dimensions, $(v, g)$, to dynamics in only one dimension, $v$, via the maximum entropy principle.

We should point out that the diffusion approximation is not necessary for deriving our main result, the kinetic Equations (2.37) in Section 2.4.4. We use it for the derivation presented here for two reasons: the first reason is that this is how we initially found the closure (2.36) leading to Equation (2.37), and the second reason is that the explicit description of the steady conductance statistics, given in Section 2.4.2, leads directly to the intuitive derivation of the closure (2.36) via the maximum entropy principle in Section 2.4.4.

The neuronal conductance jump terms, (2.3b) and (2.3c) in Equation (2.3), complicate the analysis of this equation. Recall that the sizes of these jumps are $\bar{S} / p N \sigma$ for a jump induced by a spike from another neuron, and $f / \sigma$ for a jump induced by a spike from the external input, respectively. Assuming these jumps to be small, we Taylor-expand the terms (2.3b) and (2.3c) to second order and obtain the advection-diffusion equation

$$
\partial_{t} \rho=\partial_{v}\left\{\left[\left(\frac{v-\varepsilon_{r}}{\tau}\right)+g\left(\frac{v-\varepsilon_{E}}{\tau}\right)\right] \rho\right\}+\partial_{g}\left\{\frac{1}{\sigma}(g-\bar{g}(t)) \rho+\frac{\sigma_{g}^{2}(t)}{\sigma} \partial_{g} \rho\right\},
$$


with $\bar{g}(t)$ as in Equation (2.11) and

$$
\sigma_{g}^{2}(t)=\frac{1}{2 \sigma}\left[f^{2} \nu(t)+\frac{\bar{S}^{2}}{p N} m(t)\right]
$$

valid for $\varepsilon_{r} \leq v<V_{T}$ and $-\infty<g<\infty$.

The extension of the conductance range to negative values is a consequence of this diffusion approximation; under this approximation, upward jumps in conductance at spike times are replaced by the appropriate upwards drift and also diffusion. The latter pushes conductances upwards, but downwards as well, and so they may cross into the $g<0$ region. Thus, the smallness but not necessarily vanishing of the conductance pdf, $\rho(v, g)$, for negative conductance values is in accordance with the diffusion approximation. An excessive amount of negative conductances in the solution signifies a breakdown of the applicability of the diffusion approximation (Cf. Ref. [99]).

Note that the coefficient $\sigma_{g}^{2}(t)$ describes the amount of synaptic-input fluctuations arriving at a network neuron. Note also that the ratio between the mean of the synaptic input and its fluctuations is controlled by the ratios $f / \sigma$ and $\bar{S} / p N \sigma$, i.e., the conductance jump sizes. Thus, the advection-diffusion Equation (2.22) is valid precisely in the regime when $\sigma_{g}^{2}(t) \ll \bar{g}(t)$, which is when the synaptic-input fluctuations are small compared to its mean.

Equation (2.22) can still be written in the probability conservation form (2.4), with the membrane-potential flux still given by Equation (2.5a), and conductance probability flux now given by the equation

$$
J_{G}(v, g)=-\left[\frac{1}{\sigma}(g-\bar{g}(t)) \rho(v, g)+\frac{\sigma_{g}^{2}(t)}{\sigma} \partial_{g} \rho(v, g)\right] .
$$

The voltage-flux boundary condition (2.6a) remains valid under the diffusion approximation, while the conductance boundary conditions (2.6b) and (2.6c) are best captured by the requirements that

$$
\rho(v, g \rightarrow \pm \infty) \rightarrow 0
$$

together with all its derivatives, and that $\rho(v, g<0)$ must be small, valid for $\varepsilon_{r} \leq v<$ $V_{T}$.

The equation pair (2.6a) and (2.25) constitutes the boundary conditions for the advection-diffusion Equation (2.22). The evolution of the firing rate is still determined by the self-consistency condition $(2.6 \mathrm{~d})$. The requirement that $\rho(v, g<0) \ll 1$ is an integral part of the diffusion approximation.

Since Equation $(2.22)$ is a nonlinear $(2+1)$-dimensional partial differential equation, we should be able to reduce computational cost if its dynamics can be reduced to a $(1+1)$-dimensional effective dynamics. To describe this effective dynamics, in Section 2.4.4, we derive a system of $(1+1)$-dimensional kinetic equations using a closure based on the maximum-entropy principle.

2.4.2. Dynamics of conductances. The dynamics of the conductances alone can be isolated from the membrane potential dynamics by projecting out the variable $v$ from Equation (2.22). Thus, for the conductance probability density function,

$$
\rho^{(g)}(g)=\int_{\varepsilon_{r}}^{V_{T}} \rho(v, g) d v
$$


we derive the Fokker-Planck equation

$$
\partial_{t} \rho^{(g)}(g)=\partial_{g}\left[\frac{(g-\bar{g}(t))}{\sigma} \rho^{(g)}(g)+\frac{\sigma_{g}^{2}(t)}{\sigma} \partial_{g} \rho^{(g)}(g)\right],
$$

valid for $-\infty<g<\infty$, by integrating Equation (2.22) over $v$ and using the flux condition (2.6a) at the boundary. Equation (2.27) can again be written in the conservation form, $\partial_{t} \rho^{(g)}(g, t)+\partial J_{G}(g, t)=0$, with the flux

$$
J_{G}(g)=-\left[\frac{(g-\bar{g}(t))}{\sigma} \rho^{(g)}(g)+\frac{\sigma_{g}^{2}(t)}{\sigma} \partial_{g} \rho^{(g)}(g)\right] .
$$

The boundary conditions (2.25) give two boundary conditions for Equation (2.27). The requirement that $\rho^{(g)}(g<0)$ should be small must also be satisfied.

For time-homogeneous input, when $\nu$, and so $\bar{g}$ and $\sigma_{g}^{2}$, are constant, the timeindependent solution of Equation (2.27) is given by the Gaussian

$$
\rho_{e q}^{(g)}(g)=\frac{1}{\sqrt{2 \pi} \sigma_{g}} \exp \left[-\frac{1}{2 \sigma_{g}^{2}}(g-\bar{g})^{2}\right],
$$

with the mean $\bar{g}$ and the variance $\sigma_{g}^{2}$ given by Equations (2.11) and (2.23), provided $\bar{g} \gg \sigma_{g}$, which is one of the validity conditions for the diffusion approximation, as discussed in Section 2.4.1. Since Equation (2.27) shows that the time-scale for the evolution of $\rho^{(g)}(g)$ is $\sigma$ (for sufficiently small $\sigma$ ), the solution starting with any initial condition of $\rho^{(g)}(g)$ will rapidly converge to the approximate form in Equation (2.28).

For time-dependent $\bar{g}(t)$ and $\sigma_{g}^{2}(t)$, if their time-scale is much slower than $\sigma$, then, in the limit of $\sigma \rightarrow 0$, the true density $\rho^{(g)}(g, t)$ becomes slaved to the time-invariant solution approximated by Equation (2.28). This is generally the case for AMPA conductances, which change on a much faster scale than the typical time-scales of the stimuli [63].

2.4.3. Dynamics of membrane potentials. To project out the variable $g$ from Equation (2.22) is considerably more involved than to project out the variable $v$. In fact, in the process, we are led to an infinite hierarchy of $(1+1)$-dimensional partial differential equations for the conditional conductance moments, which we must then reduce to a finite set using an appropriate closure. We derive the infinite hierarchy in the present section and leave the closure for the next section.

We begin by deriving the equations for the conditional conductance moments

$$
\mu_{0}(v)=1, \quad \mu_{n}(v)=\int_{-\infty}^{\infty} g^{n} \rho(g \mid v) d g, \quad n=1,2,3, \ldots,
$$

where

$$
\rho(g \mid v)=\frac{\rho(v, g)}{\rho^{(v)}(v)}
$$

with

$$
\rho^{(v)}(v)=\int_{-\infty}^{\infty} \rho(v, g) d g
$$


Note that we are integrating over both positive and negative conductance values in accordance with the diffusion approximation, as explained in Section 2.4.1.

First, integrating Equation (2.22) over $g$ yields the equation for the voltage probability density,

$$
\partial_{t} \rho^{(v)}(v)=\partial_{v}\left\{\left[\left(\frac{v-\varepsilon_{r}}{\tau}\right)+\mu_{1}(v)\left(\frac{v-\varepsilon_{E}}{\tau}\right)\right] \rho^{(v)}(v)\right\} .
$$

Next, multiplying Equation (2.22) by $g$ and integrating over $g$ yields the equation for the first conditional conductance moment,

$$
\begin{aligned}
\partial_{t}\left[\mu_{1}(v) \rho^{(v)}(v)\right]=- & \frac{1}{\sigma}\left[\mu_{1}(v)-\bar{g}(t)\right] \rho^{(v)}(v) \\
& +\partial_{v}\left[\left(\frac{v-\varepsilon_{r}}{\tau}\right) \mu_{1}(v) \rho^{(v)}(v)\right] \\
& +\partial_{v}\left[\left(\frac{v-\varepsilon_{E}}{\tau}\right) \mu_{2}(v) \rho^{(v)}(v)\right],
\end{aligned}
$$

where we have used Equation (2.24) and the boundary conditions (2.25) for $\rho(v, g)$ and all its derivatives. Likewise, for general $n \geq 2$, multiplying Equation (2.22) by $g^{n}$ and integrating over $g$ yields the equations for the higher conditional conductance moments,

$$
\begin{aligned}
\partial_{t}\left[\mu_{n}(v) \rho^{(v)}(v)\right]=- & \frac{n}{\sigma}\left[\mu_{n}(v)-\mu_{n-1}(v) \bar{g}(t)\right] \rho^{(v)}(v)+n(n-1) \frac{\sigma_{g}^{2}(t)}{\sigma} \mu_{n-2}(v) \rho^{(v)}(v) \\
& +\partial_{v}\left[\left(\frac{v-\varepsilon_{r}}{\tau}\right) \mu_{n}(v) \rho^{(v)}(v)\right] \\
& +\partial_{v}\left[\left(\frac{v-\varepsilon_{E}}{\tau}\right) \mu_{n+1}(v) \rho^{(v)}(v)\right] .
\end{aligned}
$$

We can simplify Equations (2.31) using Equation (2.30). In particular, we restate Equation (2.31a) in the form

$$
\begin{aligned}
\partial_{t} \mu_{1}(v)=- & \frac{1}{\sigma}\left[\mu_{1}(v)-\bar{g}(t)\right]+\left[\left(\frac{v-\varepsilon_{r}}{\tau}\right)+\mu_{1}(v)\left(\frac{v-\varepsilon_{E}}{\tau}\right)\right] \partial_{v} \mu_{1}(v) \\
& +\frac{1}{\rho^{(v)}(v)} \partial_{v}\left[\left(\frac{v-\varepsilon_{E}}{\tau}\right)\left[\mu_{2}(v)-\mu_{1}^{2}(v)\right] \rho^{(v)}(v)\right],
\end{aligned}
$$

and Equation (2.31b) in the form

$$
\begin{aligned}
\partial_{t} \mu_{n}(v)=- & \frac{1}{\sigma}\left[\mu_{n}(v)-\bar{g}(t) \mu_{n-1}(v)\right]+\frac{n(n-1) \sigma_{g}^{2}(t)}{\sigma} \mu_{n-2}(v) \\
& +\left[\left(\frac{v-\varepsilon_{r}}{\tau}\right)+\mu_{1}(v)\left(\frac{v-\varepsilon_{E}}{\tau}\right)\right] \partial_{v} \mu_{n}(v) \\
& +\frac{1}{\rho^{(v)}(v)} \partial_{v}\left[\left(\frac{v-\varepsilon_{E}}{\tau}\right)\left[\mu_{n+1}(v)-\mu_{n}(v) \mu_{1}(v)\right] \rho^{(v)}(v)\right] .
\end{aligned}
$$

Equations (2.30) and (2.32) give the infinite hierarchy of $(1+1)$-dimensional partial differential equations for the voltage probability density $\rho^{(v)}(v)$ and the conditional conductance moments $\mu_{n}(v), n=1,2,3, \ldots$, referred to at the beginning of this section. Truncating this hierarchy by using an appropriate closure will be the task of the next section. 
2.4.4. Closure via the maximum entropy principle. Equations $(2.30)$ and (2.32) imply that the evolution of the voltage probability density function $\rho^{(v)}(v)$ depends on the first conditional moment $\mu_{1}(v)$, the evolution of $\mu_{1}(v)$ depends on the second conditional moment $\mu_{2}(v)$, and so on. The desired dimension reduction of Equation $(2.22)$ to a $(1+1)$-dimensional system is accomplished by closing this infinite hierarchy of equations (2.30) and (2.32) at a finite order in such a way that the truncated dynamics still capture the essential behavior of the I\&F system (2.1).

We postulate a closure based on the maximum entropy principle, and close Equation $(2.32 \mathrm{a})$ by approximating the second conditional moment $\mu_{2}(v)$ as a function of the first conditional moment $\mu_{1}(v)$. In the steady state, when $\bar{g}$ and $\sigma_{g}^{2}$ are constants, the entropy

$$
S[\rho] \equiv-\int_{-\infty}^{\infty} \rho(g \mid v) \log \frac{\rho(g \mid v)}{\rho_{e q}^{(g)}(g)} d g,
$$

where $\rho_{e q}^{(g)}(g)$ is the steady solution (2.28) of Equation (2.27), is maximized subject to the natural constraints

$$
\begin{aligned}
& \text { (i) } \int_{-\infty}^{\infty} \rho(g \mid v) d g=1, \\
& \text { (ii) } \int_{-\infty}^{\infty} g \rho(g \mid v) d g=\mu_{1}(v) .
\end{aligned}
$$

Intuitively, one would anticipate the entropy $S[\rho]$ in Equation (2.33) to be maximized for the following reason: as discussed in Section 2.4.2, when the diffusion approximation holds and for sufficiently short conductance time-scale $\sigma$, the conductance probability density rapidly relaxes towards the equilibrium density $\rho_{e q}^{(g)}(g)$ regardless of the membrane-potential values. Therefore, we should expect that the conditional distribution $\rho(g \mid v)$ will rather closely follow the shape of the equilibrium distribution $\rho_{e q}^{(g)}(g)$ along the actual trajectory of the network, indicating that the entropy $S[\rho]$ should be maximal along this trajectory. We remark that similar maximum-entropy principles have been employed to find approximate solutions in fluid mechanics $[2,71]$.

The above constrained entropy maximization yields the solution

$$
\hat{\rho}(g \mid v)=\rho_{e q}(g) \exp \left[-\lambda_{0}(v)-\lambda_{1}(v) g\right],
$$

where $\lambda_{0}(v)$, and $\lambda_{1}(v)$ are the corresponding Lagrange multipliers. Solving the constraints (i) and (ii) shows that the entropy attains its maximum at the function of the form

$$
\hat{\rho}(g \mid v)=\frac{1}{\sqrt{2 \pi} \sigma_{g}} \exp \left\{-\frac{\left[g-\mu_{1}(v)\right]^{2}}{2 \sigma_{g}^{2}}\right\},
$$

with $\mu_{1}(v)=\bar{g}-\lambda_{1}(v) \sigma_{g}^{2}$. We then use this entropy-maximizing solution $\hat{\rho}(g \mid v)$ to evaluate the equation $\mu_{2}(v)=\int_{-\infty}^{\infty} g^{2} \hat{\rho}(g \mid v) d g$ and thus express the second moment $\mu_{2}(v)$ in terms of the first moment $\mu_{1}(v)$. In this way, we derive the closure condition

$$
\mu_{2}(v)=\mu_{1}^{2}(v)+\sigma_{g}^{2} .
$$

In the time-dependent situation, the closure in Equation (2.35) can be extended to the case when the external input rate $\nu(t)$ varies slowly compared to the timescale determined by the conductance decay constant $\sigma$. In this case, as discussed in 
Section (2.4.2), the conductance dynamics will rapidly relax to the quasi-stationary distribution (2.28). Therefore, we can postulate again the same closure as in Equation $(2.35)$,

$$
\mu_{2}(v, t)=\mu_{1}^{2}(v, t)+\sigma_{g}^{2}(t),
$$

to capture the time-dependent dynamics.

Under the closure (2.36), Equations (2.30) and (2.31a) become closed with respect to the density $\rho^{(v)}(v)$ and first moment $\mu_{1}(v)$, and we arrive at the $(1+1)$-dimensional kinetic equations governing the coupled excitatory neuronal networks:

$$
\begin{aligned}
& \partial_{t} \rho^{(v)}(v)=\partial_{v}\left\{\left[\left(\frac{v-\varepsilon_{r}}{\tau}\right)+\mu_{1}(v)\left(\frac{v-\varepsilon_{E}}{\tau}\right)\right] \rho^{(v)}(v)\right\}, \\
& \partial_{t} \mu_{1}(v)=-\frac{1}{\sigma}\left[\mu_{1}(v)-\bar{g}(t)\right]+\frac{\sigma_{g}^{2}(t)}{\rho^{(v)}(v)} \partial_{v}\left[\left(\frac{v-\varepsilon_{E}}{\tau}\right) \rho^{(v)}(v)\right] \\
&+\left[\left(\frac{v-\varepsilon_{r}}{\tau}\right)+\mu_{1}(v)\left(\frac{v-\varepsilon_{E}}{\tau}\right)\right] \partial_{v} \mu_{1}(v) .
\end{aligned}
$$

Note that Equation (2.37a) can be written in the flux form $\partial_{t} \rho^{(v)}(v)+\partial_{v} \mathcal{J}_{V}(v, t)=0$, with the probability flux

$$
\mathcal{J}_{V}(v, t)=-\left[\left(\frac{v-\varepsilon_{r}}{\tau}\right)+\mu_{1}(v)\left(\frac{v-\varepsilon_{E}}{\tau}\right)\right] \rho^{(v)}(v) .
$$

Using the definitions (2.29), we find that the flux $\mathcal{J}_{V}(v, t)$ equals the $g$-integral of the flux $J_{V}(v, g, t)$ in Equation (2.5a), i.e.,

$$
\mathcal{J}_{V}(v, t)=\int_{-\infty}^{\infty} J_{V}(v, g, t) d g
$$

We remark that, mathematically, a closure is frequently related to coarse-graining, in particular, to the question whether there exists a scale below which the dynamics is either sufficiently smooth and featureless by itself or else can be averaged out. In [32], the closure (2.36) was postulated from this coarse-graining viewpoint, in particular, by assuming the conditional variance $\Sigma^{2}(v)=\mu_{2}(v)-\mu_{1}^{2}(v)$ to be a sufficiently slowly varying function of the membrane potential $v$.

2.4.5. Boundary conditions. The boundary conditions for the kinetic Equation (2.37) can be derived from the boundary condition (2.6a) for the voltage probability flux $J_{V}(v, g)$ in Equation (2.5a) and the closure (2.36), in particular, by considering the boundary values at $v=\varepsilon_{r}$ and $v=V_{T}$ of the $g$-integral and moments of $J_{V}(v, g)$.

First, integrating $J_{V}(v, g)$ over the $g$-range and using Equations (2.6d) and (2.39), we find

$$
\mathcal{J}_{V}\left(V_{T}, t\right)=\mathcal{J}_{V}\left(\varepsilon_{r}, t\right)=m(t)
$$

i.e., the population-averaged firing rate per neuron, $m(t)$, must equal the total voltage flux, $\mathcal{J}_{V}$ in Equation (2.38), at the threshold and reset. This condition can be rewritten using Equation (2.38) as

$$
\left[\left(V_{T}-\varepsilon_{r}\right)+\left(V_{T}-\varepsilon_{E}\right) \mu_{1}\left(V_{T}\right)\right] \rho^{(v)}\left(V_{T}\right)=\left(\varepsilon_{r}-\varepsilon_{E}\right) \mu_{1}\left(\varepsilon_{r}\right) \rho^{(v)}\left(\varepsilon_{r}\right)=-\tau m(t)
$$



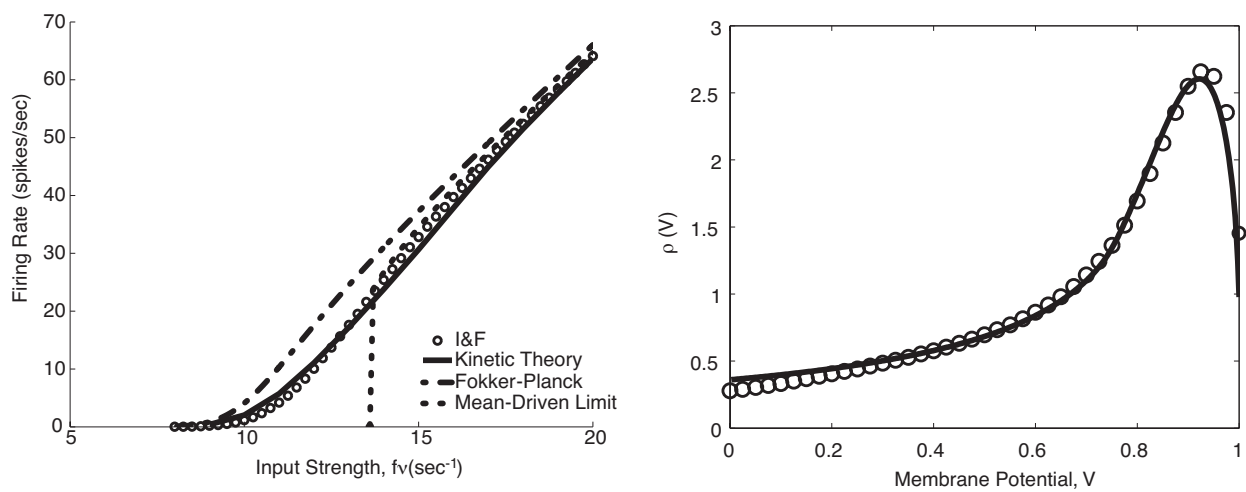

FIG. 2.2. Comparison between the predictions of the kinetic theory (2.37) and full numerical simulations of the IEFF network (2.1). Left: The average population firing rate per neuron, $m$, as a function of the average external-input conductance $f \nu$ for the full simulations (solid), kinetic theory (dashed), Fokker-Planck equation (dash-dotted), and the mean-field theory (dotted). Right: The membrane potential distribution $\rho^{(v)}(v)$. The solid curve represents the results of the IEGF network simulation and the circles the kinetic theory. [Reproduced with permission from Ref. [32], (Copyright 2004, by National Academy of Sciences, USA).J

Furthermore, for the first $g$-moment of the flux $J_{V}(v, g)$,

$$
\eta(v)=\int_{-\infty}^{\infty} g J_{V}(v, g) d g
$$

using the closure (2.36), we derive the expression

$$
\eta(v)=\mathcal{J}_{V}(v, t) \mu_{1}(v)-\sigma_{g}^{2}\left(\frac{v-\varepsilon_{E}}{\tau}\right) \rho^{(v)}(v),
$$

for which the boundary condition (2.6a) implies the periodic boundary condition

$$
\eta\left(V_{T}\right)=\eta\left(\varepsilon_{r}\right),
$$

i.e.,

$$
\begin{aligned}
& \mathcal{J}_{V}\left(V_{T}, t\right) \mu_{1}\left(V_{T}\right)-\sigma_{g}^{2}\left(\frac{V_{T}-\varepsilon_{E}}{\tau}\right) \rho^{(v)}\left(V_{T}\right) \\
= & \mathcal{J}_{V}\left(\varepsilon_{r}, t\right) \mu_{1}\left(\varepsilon_{r}\right)-\sigma_{g}^{2}\left(\frac{\varepsilon_{r}-\varepsilon_{E}}{\tau}\right) \rho^{(v)}\left(\varepsilon_{r}\right) .
\end{aligned}
$$

Using Equation (2.40), we hence derive the second boundary condition,

$$
\tau m(t)\left[\mu_{1}\left(V_{T}\right)-\mu_{1}\left(\varepsilon_{r}\right)\right]=\sigma_{g}^{2}\left[\left(V_{T}-\varepsilon_{E}\right) \rho^{(v)}\left(V_{T}\right)-\left(\varepsilon_{r}-\varepsilon_{E}\right) \rho^{(v)}\left(\varepsilon_{r}\right)\right]
$$

for the kinetic Equations (2.37). We have thus found that Equations (2.41) constitute the nonlinear boundary conditions for the kinetic Equations (2.37).

As can be seen from Figures 2.2 and 2.3, the kinetic Equation (2.37), derived under the closure (2.36), and their nonlinear boundary conditions (2.41), capture the one-point statistical properties of the I\&F neuronal network (2.1) dynamics very well for both steady and time-dependent external-driving Poisson rates. Moreover, solving these equations is $\sim 10^{4}$ to $10^{5}$ times faster than using direct I\&F dynamics simulations to obtain the firing rates computationally $[31,32,96]$. 

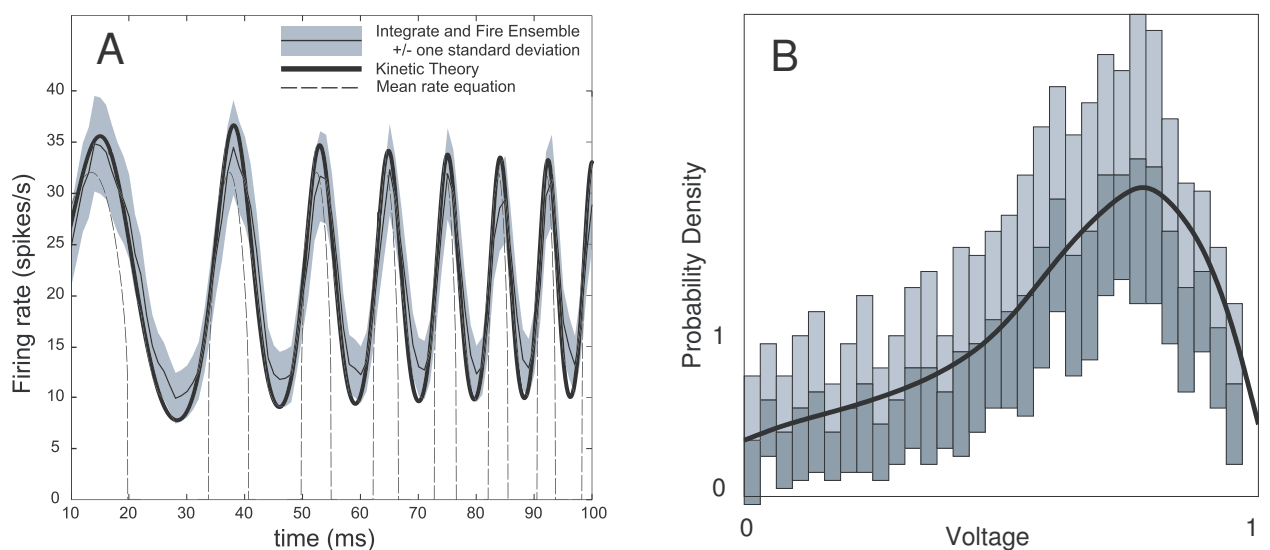

FIG. 2.3. Dynamical accuracy of the kinetic theory in fluctuation-driven dynamics for a network (2.1) of purely excitatory IEF neurons driven by the time-varying input rate $\nu(t)=$ $\nu_{a} \exp \left[\omega \sin \left(2 \pi t / s+(2 \pi t / s)^{2}\right)\right]$. Left: The firing rate $m(t)$. Thin solid line: simulation result averaged over an ensemble of $10^{4}$ identically structured networks. The upper and lower boundaries of the gray area mark the values one-standard deviation away from the mean. Thick solid line: Kinetic theory (2.37). Dashed line: Mean-driven limit (2.10); Right: An instantaneous probability density function of the membrane potential. The upper and lower boundaries of the gray area mark the values one-standard deviation away from the mean, measured from the ensemble of the $10^{4}$ networks. Thick solid line: Kinetic theory (2.37). [Reproduced with permission from Ref. [31], (Copyright 2006, by International Press).]

2.4.6. Mean-driven limit of the kinetic equations. We now show that the mean-field system (2.10) follows from the kinetic Equation (2.37) in the limit of vanishing synaptic-input fluctuations, $\sigma_{g} \rightarrow 0$, under the assumption (2.8). In particular, under this assumption, Equations (2.29) and (2.9) imply that $\mu_{1}(v)=$ $\langle g\rangle$, so that Equations (2.10) follow after letting $\sigma_{g} \rightarrow 0$ in Equation (2.37b). The developments of the rest of Section 2.3 now follow immediately. Note that the meanfield system (2.10) was derived from a more general framework, i.e., without the diffusion approximation, and as we will see in the next section, one can do the same for a slight generalization of the system of kinetic Equation (2.37).

For small values of synaptic-input fluctuations, $\sigma_{g} \ll \bar{g}$, the comparison in Figure 2.2 of the approximate gain curve (2.17) with the corresponding numerical solutions of the kinetic Equation (2.37) and also the I\&F system (2.1) shows good agreement along the upper, stable branch of this curve, that is, in the mean-driven regime. Recall that from Equation (2.23), it follows that $\sigma_{g} \ll \bar{g}$ is equivalent to small conductance jumps, $f / \sigma$ and $\bar{S} / p N \sigma$, for which the diffusion approximation (2.22) is valid. Note, in Figure 2.3, that the mean-field Equation (2.10) misrepresents low firing rates as vanishing.

2.4.7. Generalizations of the kinetic equations. As mentioned in Section 2.4.1, a slight generalization of the kinetic Equation (2.37) can be derived directly from the Boltzmann Equation (2.3) without resorting to the diffusion approximation (2.22). In particular, as shown in [69], a hierarchy of conditional moment equations very similar to Equations (2.30) and (2.32), as presented in Section 2.4.3, can be derived straight from the Boltzmann Equation (2.3). The closure (2.36) must be 
replaced by the closure

$$
\mu_{2}(v, t)-\mu_{1}^{2}(v, t)=\left\langle g^{2}\right\rangle(t)-[\langle g\rangle(t)]^{2},
$$

i.e., the substitution of the unconditional conductance variance for its conditional variance. In the resulting system of kinetic equations analogous to Equation (2.37), the mean and variance of the synaptic input, $\bar{g}(t)$ and $\sigma_{g}^{2}(t)$, must also be replaced by the mean neuronal conductance, $\langle g\rangle(t)$, and its variance, $\left\langle g^{2}\right\rangle(t)-[\langle g\rangle(t)]^{2}$. The dynamics of $\langle g\rangle(t)$ are described by Equation (2.11). To find the dynamics of $\left\langle g^{2}\right\rangle(t)$, one proceeds in a manner similar to that presented in Section 2.4.2 to eliminate the voltages from Equation (2.3) and then derive a closed equation for $\left\langle g^{2}\right\rangle(t)$ from the resulting differential-difference equation. The final equation for the second moment $\left\langle g^{2}\right\rangle(t)$ is

$$
\frac{d}{d t}\left\langle g^{2}\right\rangle=-\frac{2}{\sigma}\left[\left\langle g^{2}\right\rangle-\langle g\rangle \bar{g}(t)-\sigma_{g}^{2}(t)\right]
$$

The kinetic Equations (2.37) remain valid approximately at time-scales larger than $\mathcal{O}(\sigma)$, the conductance time scale. In particular, Equations (2.11) and (2.43) imply that, for time-independent external drive, the mean and variance of the conductances relax to the mean and variance of the synaptic input, $\bar{g}$ and $\sigma_{g}^{2}$, respectively, so that Equations (2.37) become the exact limit of the more general kinetic equations in this case.

Extension of the kinetic theory, described in the previous sections, to networks that include both excitatory and inhibitory neurons, as well as simple and complex cells, presents few conceptual obstacles, and is outlined in $[31,95]$. When including two types of receptors that bind the same neurotransmitter, such as AMPA and NMDA, but respond on different time-scales, it is important to keep in mind the fact that these receptors are activated by (parts of) the same spike train [99]. This leads to correlations in the postsynaptic conductances, which are expressed by mixed second-derivative terms in the drift-diffusion equation analogous to Equation (2.22). In addition, for sufficiently disparate conductance time scales, the analog of the closure (2.42) must be used instead of (2.36), and equations analogous to the evolution equations for the conductance moments, (2.11) and (2.43), must be added to the three kinetic partial differential equations. Further extensions include the incorporation of a refraction period and higher-order conductance kinetics.

2.5. Fokker-Planck description of neuronal network dynamics. A number of neuroscience studies address statistical properties of neuronal network dynamics using the Fokker-Planck Equation [1,23-25,65,81,105]. These studies typically assume that the neuronal conductances can be described as white noise, and consequently the neuronal voltages as an Ornstein-Uhlenbeck-like process [49]. Here, we discuss our recent work on the bifurcations of steady-state gain curves as described by the Fokker-Planck Equation [65], as well as the mutual interconnections among the kinetic-theoretic, Fokker-Planck, and mean-field descriptions. In particular, we discuss how the Fokker-Planck description captures the voltage pdfs and gain curves in the fluctuation-driven regime quite well, while it is in fact less accurate in the mean-driven regime than the simpler mean-field limit.

2.5.1. Fokker-Planck description in the case of vanishing conductance time scale. In the limit of infinitely-fast conductance time-scale, $\sigma \rightarrow 0$, Equation $(2.1 \mathrm{~b})$ shows that the neuronal conductances are slaved to the synaptic input. 
Therefore, the $i$ th neuron's conductance becomes

$$
G_{i}(t)=f \sum_{\mu} \delta\left(t-t_{\mu}^{i}\right)+\frac{S}{N} \sum_{j \neq i} \sum_{\mu} p_{i j \mu} \delta\left(t-t_{j \mu}\right)
$$

where, as in Section 2.1, $t_{\mu}^{i}$ are the spike-times of the external input to the $i$ th neuron, $t_{j \mu}$ the spike-times of the network neurons, and $p_{i j \mu}$ the Bernoulli random variable describing the synaptic release probability. Equation (2.1a) for the $i$ th neuronal voltage in this limit thus reduces to the form

$$
\tau \frac{d V_{i}}{d t}=-\left(V_{i}-\varepsilon_{r}\right)-\left[f \sum_{\mu} \delta\left(t-t_{\mu}^{i}\right)+\frac{S}{N} \sum_{j \neq i} \sum_{\mu} p_{i j \mu} \delta\left(t-t_{j \mu}\right)\right]\left(V_{i}-\varepsilon_{E}\right),
$$

for $i=1, \ldots, N$. The spiking and reset mechanism operates as for the original network (2.1).

In-between spikes, the $i$ th neuron's voltage in Equation (2.44) decays towards reset, while at each time of a received spike it jumps. Its values immediately before and after the jump are easily seen to be connected by the relations

$$
V_{i}\left(\tau_{i j}^{+}\right)=(1-\Gamma) V_{i}\left(\tau_{i j}^{-}\right)+\Gamma \varepsilon_{E}, \quad \Gamma=1-e^{-f / \tau}
$$

in the case of an external-input spike, and

$$
V_{i}\left(t_{k l}^{+}\right)=(1-\Sigma) V_{i}\left(t_{k l}^{-}\right)+\Sigma \varepsilon_{E}, \quad \Sigma=1-e^{-\bar{S} / p N \tau}
$$

in the case of a network-neuron spike, provided that $V_{i}\left(\tau_{i j}^{+}\right), V_{i}\left(t_{k l}^{+}\right)<V_{T}$, respectively $[31,87]$. Here, as in Section $2.2, \bar{S}$ is defined as $\bar{S}=p S$, where $p$ is the synaptic release probability.

The limiting system (2.44) exhibits a peculiar type of dynamical behavior due to its instantaneous conductance scale. In particular, since a neuron's voltage can only rise when it receives a spike, neurons must fire in instantaneous cascades. Each cascade starts with an external-driving spike to a network neuron, which makes this neuron's voltage jump over the firing threshold, $V_{T}$. The resulting spike makes the voltages of other neurons in the network jump, pushing those that were high enough over $V_{T}$ and thus making the corresponding neurons fire. This process continues until all the neurons with sufficiently high voltages have fired. For sufficiently high coupling strengths, such cascades may exhaust the entire network and result in periodic "total firing events" $[80,81]$. For the network to instead maintain an asynchronous firing regime, such that the total network spike output can be assumed as a Poisson spike train and that this train is approximately independent of the external-drive spike trains driving the individual network neurons, we must again assume high externalinput Poisson rate, $\nu(t) \gg 1 / \tau$, large network size, $N \gg 1$, and small spike strength, $f / \tau, \bar{S} / p N \tau \ll 1$. These assumptions are similar to those that lead to the diffusion approximation in Section 2.4 .

A derivation analogous to that presented in Section 2.2 leads to the Boltzmann equation for the neuronal-voltage pdf, $\rho^{(v)}(v, t)$, corresponding to the network (2.44), which is

$$
\begin{aligned}
\partial_{t} \rho^{(v)}(v, t)=\partial_{v} & {\left[\left(\frac{v-\varepsilon_{r}}{\tau}\right) \rho^{(v)}(v, t)\right]+\nu(t)\left[\frac{1}{1-\Gamma} \rho^{(v)}\left(\frac{v-\Gamma \varepsilon_{E}}{1-\Gamma}, t\right)-\rho^{(v)}(v, t)\right] } \\
& +N p m(t)\left[\frac{1}{1-\Sigma} \rho^{(v)}\left(\frac{v-\Sigma \varepsilon_{E}}{1-\Sigma}, t\right)-\rho^{(v)}(v, t)\right]
\end{aligned}
$$


with the coefficients $\Gamma$ and $\Sigma$ as in Equations (2.45a) and (2.45b).

Equation (2.46) can again be written in the form of probability conservation, $\partial_{t} \rho(v, t)+\partial_{v} J_{V}^{b}(v, t)=0$, with the probability flux

$$
\begin{aligned}
J_{V}^{b}(v, t)=- & \left(\frac{v-\varepsilon_{r}}{\tau}\right) \rho^{(v)}(v, t)+\nu(t) \int_{\frac{v-\Gamma \varepsilon_{E}}{1-\Gamma}}^{v} \rho^{(v)}(u, t) d u \\
& +N p m(t) \int_{\frac{v-\Sigma \varepsilon_{E}}{1-\Sigma}}^{v} \rho^{(v)}(u, t) d u .
\end{aligned}
$$

Here, the first term on the right-hand side describes the flux due to the smooth streaming of phase points under the relaxation dynamics in Equation (2.44), and the last two the flux due to the voltage jumps induced by the external-input and network spikes.

The first term in Equation (2.47) is clearly nonpositive, signifying downwards relaxation of the neuronal voltages. At the firing threshold, $V_{T}$, this relaxation cannot take place since any neuron's voltage that crosses $V_{T}$ is immediately returned to the reset $\varepsilon_{r}$, and so the term describing it must vanish. The only way in which this can happen is if

$$
\rho^{(v)}\left(V_{T}, t\right)=0
$$

which gives one boundary condition for Equation (2.44). Equating the probability flux across the reset, $\varepsilon_{r}$, and threshold, $V_{T}$, with the firing rate,

$$
\begin{aligned}
m(t) & =J_{V}^{b}\left(\varepsilon_{r}, t\right)=J_{V}^{b}\left(V_{T}, t\right) \\
& =\nu(t) \int_{\frac{V_{T}-\Gamma \varepsilon_{E}}{1-\Gamma}}^{V_{T}} \rho^{(v)}(u, t) d u+N p m(t) \int_{\frac{V_{T}-\Sigma \varepsilon_{E}}{1-\Sigma}}^{V_{T}} \rho^{(v)}(u, t) d u,
\end{aligned}
$$

gives the second boundary condition.

Assuming small jumps, $f / \tau, \bar{S} / N p \ll 1$, we Taylor-expand the right-hand side of the Boltzmann Equation (2.44), i.e., make the diffusion approximation, to find the Fokker-Planck equation

$$
\partial_{t} \rho^{(v)}(v)=\partial_{v}\left\{\left[\left(\frac{v-\varepsilon_{r}}{\tau}\right)+\gamma(t)\left(\frac{v-\varepsilon_{E}}{\tau}\right)\right] \rho^{(v)}(v)+q^{2}(t) \frac{\left(v-\varepsilon_{E}\right)^{2}}{\tau} \partial_{v} \rho^{(v)}(v)\right\}
$$

where

$$
\gamma(t)=\bar{g}(t)+q^{2}(t)
$$

with $\bar{g}(t)$ as in Equation (2.11) and

$$
q^{2}(t)=\frac{1}{2 \tau}\left[f^{2} \nu(t)+\frac{\bar{S}^{2}}{p N} m(t)\right] .
$$

The corresponding probability flux is

$$
J_{V}^{f p}(v, t)=-\left[\left(\frac{v-\varepsilon_{r}}{\tau}\right)+\gamma(t)\left(\frac{v-\varepsilon_{E}}{\tau}\right)\right] \rho^{(v)}(v)-q^{2}(t) \frac{\left(v-\varepsilon_{E}\right)^{2}}{\tau} \partial_{v} \rho^{(v)}(v),
$$


and the boundary conditions are (2.48) and

$$
J_{V}^{f p}\left(\varepsilon_{r}, t\right)=J_{V}^{f p}\left(V_{T}, t\right)=m(t) .
$$

Note that, formally,

$$
q^{2}(t)=\frac{\sigma \sigma_{g}^{2}(t)}{\tau},
$$

with $\sigma_{g}^{2}(t)$ measuring the amount of synaptic-input fluctuations, as defined in Equation $(2.23)$. Note also that the above diffusion approximation leading to the FokkerPlanck Equation (2.50) is valid when

$$
q^{2}(t) \ll \bar{g}(t),
$$

which is the small-fluctuation regime of Equation (2.50).

\subsubsection{Fokker-Planck description as a limit of the kinetic theory.}

As we have seen in the previous section, the limit $\sigma \rightarrow 0$ is highly singular for the I\&F network (2.1), as the conductances in Equation (2.1b) become a train of delta spikes, and the neuronal membrane potentials jump instead of the conductances upon receiving a spike. Nevertheless, we now show that the Fokker-Planck Equation (2.50) for $\rho^{(v)}(v)$, the probability density of the membrane potentials alone, can be derived from the kinetic Equation (2.37) in this limit in a smooth fashion.

The crucial point in this derivation is the observation that

$$
\sigma \sigma_{g}^{2}(t)=\tau q^{2}(t)=\frac{1}{2}\left[f^{2} \nu(t)+\frac{\bar{S}^{2}}{N p} m(t)\right] \sim \mathcal{O}(1)
$$

even for $\sigma \rightarrow 0$. Therefore, Equation (2.37b) in this limit reduces to

$$
\mu_{1}(v)=\bar{g}(t)+\frac{\sigma \sigma_{g}^{2}}{\tau}+\frac{\sigma \sigma_{g}^{2}}{\rho^{(v)}(v)}\left(\frac{v-\varepsilon_{E}}{\tau}\right) \partial_{v} \rho^{(v)}(v),
$$

and inserting Equation (2.56) into Equation (2.37a), together with Equation (2.54), yields the Fokker-Planck Equation (2.50). The $\sigma \rightarrow 0$ limit of the boundary conditions (2.41a) and (2.41b) implies the boundary conditions (2.53) and

$$
\left(V_{T}-\varepsilon_{E}\right) \rho^{(v)}\left(V_{T}\right)=\left(\varepsilon_{r}-\varepsilon_{E}\right) \rho^{(v)}\left(\varepsilon_{r}\right)
$$

for the Fokker-Planck Equation (2.50).

Note that the boundary condition (2.57) is different from the condition (2.48), which we have derived directly from the limiting network Equation (2.44) as $\sigma \rightarrow 0$. The two different boundary conditions, (2.57) and (2.48), reflect the non-commuting of two different limits: vanishing synaptic-input fluctuations and vanishing conductance time-scale. In most cases, these two boundary conditions produce very similar solutions, so that for modeling qualitative statistical behavior either boundary condition can be used, as shown in Figure 2.4.

Note also that the conductance jumps even near the $\sigma \rightarrow 0$ limit are never small, so the conductance-diffusion approximation leading to the advection-diffusion Equation (2.22), as well as Equation (2.22) itself, lose their meaning. The smooth reduction of the kinetic Equation (2.37) to the Fokker-Planck Equation (2.50) thus appears to be a consequence of the fact, described in Section 2.4.7, that the conductance diffusion approximation is not needed in the derivation of the kinetic equations. 


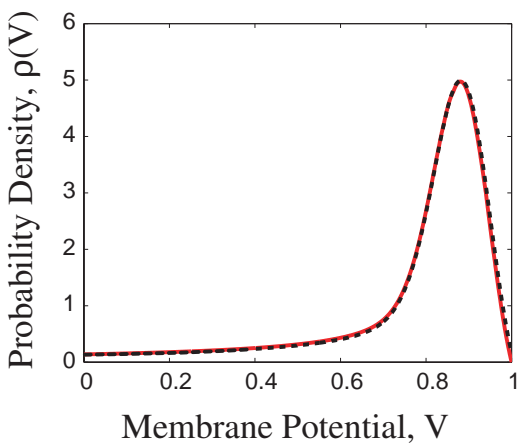

FIG. 2.4. Comparison of voltage pdfs satisfying the Fokker-Planck Equation (2.50) with the boundary conditions (2.48) (solid gray line, red online) and (2.57) (dashed line). [Reproduced with permission from Ref. [31], (Copyright 2006, by International Press).]

2.5.3. Steady solution of the Fokker-Planck equation. In the steady case, we can integrate the Fokker-Planck Equation (2.50) in $v$ and, using the flux boundary condition (2.53), obtain the first-order, linear differential equation

$$
\left[\left(v-\varepsilon_{r}\right)+\gamma\left(v-\varepsilon_{E}\right)\right] \rho^{(v)}(v)+q^{2}\left(v-\varepsilon_{E}\right)^{2} \partial_{v} \rho^{(v)}(v)=-\tau m
$$

with $\gamma$ and $q^{2}$ as in Equation (2.51). Either of the boundary conditions, (2.48) or (2.57), can remain as the sole boundary condition of Equation (2.58).

To solve Equation (2.58), it is best to use the new independent variable

$$
x=\frac{\varepsilon_{E}-\varepsilon_{r}}{\varepsilon_{E}-v},
$$

and the new dependent variable $\varrho(x)$, defined through the equation

$$
\rho^{(v)}(v)=\frac{x^{2} \varrho(x)}{\varepsilon_{E}-\varepsilon_{r}} .
$$

Note that the transformation (2.59) maps the reset potential $v=\varepsilon_{r}$ into $x=1$ and the firing threshold $v=V_{T}$ into $x=1+\bar{g}_{0}$, with $\bar{g}_{0}$ as defined in Equation (2.15). Note also that the new pdf $\varrho(x)$ was chosen so that the equation $\varrho(x) d x=\rho(v) d v$ holds. Equation (2.58) under these variable changes becomes

$$
q^{2} x^{2} \varrho^{\prime}+x\left(x+q^{2}-1-\bar{g}\right) \varrho=-m \tau,
$$

where the prime denotes differentiation upon $x$.

The boundary condition (2.48) becomes

$$
\varrho\left(1+\bar{g}_{0}\right)=0,
$$

the boundary condition (2.48) becomes

$$
\varrho(1)=\left(1+\bar{g}_{0}\right) \varrho\left(1+\bar{g}_{0}\right),
$$

and the normalization condition (2.16) becomes

$$
\int_{1}^{1+\bar{g}_{0}} \varrho(x) d x=1 .
$$


The exact solution of Equation (2.61), satisfying the condition (2.62), is given by

$$
\varrho(x) \equiv \varrho_{0}(x)=\frac{m \tau}{q^{2}} x^{(1+\bar{g}) / q^{2}-1} e^{-x / q^{2}} \int_{x}^{1+\bar{g}_{0}} s^{-(1+\bar{g}) / q^{2}-1} e^{s / q^{2}} d s .
$$

Using Equation (2.65), we find the solution of Equation (2.61) satisfying the condition (2.63) to be

$$
\varrho(x)=\varrho_{0}(x)+\frac{\varrho_{0}(1) \varrho_{H}(x)}{\left(1+\bar{g}_{0}\right) \varrho_{H}\left(1+\bar{g}_{0}\right)-\varrho_{H}(1)},
$$

where

$$
\varrho_{H}(x)=x^{(1+\bar{g}) / q^{2}-1} e^{-x / q^{2}} .
$$

The $m-f \nu$ gain curves are found from the nonlinear equation obtained using the normalization condition (2.64) and the definitions (2.11) and (2.51b) of the coefficients $\bar{g}$ and $q^{2}$, respectively. Because the voltage pdfs and gain curves produced by the solutions (2.65) and (2.66) are quite similar (Cf. Figure 2.4), we from now on focus only on those corresponding to (2.65).
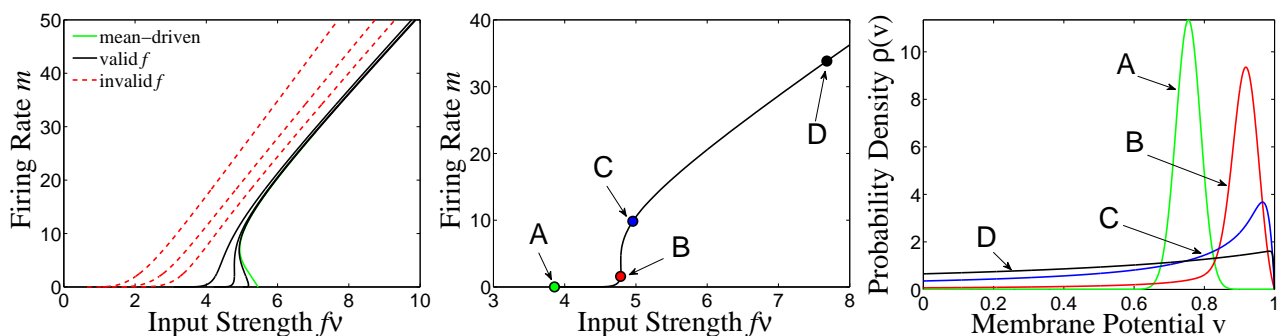

FIG. 2.5. Fokker-Planck gain curves representing the dependence of the firing rate $m$ on the external-drive strength $f \nu$. Left: The external spike strength $f$ decreases and network size $N$ increases from the left to the right curve, effectively decreasing the amount of synaptic-inputs fluctuations to zero. The strength $f$ is too large along the left three curves for the diffusion approximation, and so the Fokker-Planck Equation (2.50) ceases to be valid. Right and Middle: Voltage pdfs and their locations along a gain curve. Note the shape change from bell-shaped in the fluctuation-driven regime to increasing, except near $v=V_{T}$, in the mean-driven regime. [Reproduced with permission from Ref. [65], (Copyright 2009, by APS).]

As we can see from the left panel in Figure 2.5, in the limit of small fluctuations, $f \ll 1$ and $N \gg 1$, the gain curve obtained from the Fokker-Planck Equation (2.50) is well approximated by its mean-field limit, except near the smoothed-out corner close to the point $(f \nu, m)=\left(\bar{g}_{0}, 0\right)$, and also exhibits bistability. For increasing fluctuation values, the interval of bistability becomes narrower and eventually disappears. Moreover, the gain curves appear to approach linear asymptotes at large external-drive strengths $f \nu$, while for small $f \nu$, they appear to decay rapidly. The right two panels of Figure 2.5 indicate the shape changes of the voltage pdf, $\rho^{(v)}(v)$, between the fluctuation- and mean-driven regimes. As we will see in the next section, in these two regimes, both the voltage pdfs and gain curves can be approximated by elementary functions away from the bistability region. 
2.5.4. Mean-field limit of the Fokker-Planck solutions. To find how the steady solutions of the Fokker-Planck Equation (2.50) limit onto the corresponding steady solutions of the mean-field Equation (2.10), we recall that Equation (2.50) is only valid in the small-fluctuation limit, $q^{2} \ll \bar{g}$, imposed by the diffusion approximation. In the limit $q^{2} \rightarrow 0$, the derivative term in the steady Fokker-Planck ordinary differential Equation (2.58) vanishes, and so this equation immediately reduces to the mean-field voltage pdf in Equation (2.14), provided the firing rate $m$ does not vanish. If the firing rate $m$ does vanish, the same limit gives $\rho^{(v)}(v)=0$ pointwise for all voltage values; this contradicts the fact that $\rho^{(v)}(v)$ is a pdf and thus normalizes to unity. Therefore, one should expect the delta-function solution (2.19) in this limit, which we confirm below. We should remark that the time-dependent Fokker-Planck Equation (2.50) does not limit onto the full time-dependent mean-field system (2.10), but instead a reduced system with Equation (2.10b) replaced by the asymptotic meanconductance value $\langle g\rangle(t)=\bar{g}(t)$. This is in agreement with the $\sigma \rightarrow 0$ limit of the vanishing conductance time-scale.

To better understand the $q^{2} \rightarrow 0$ limit of the solutions of the steady Fokker-Planck Equation (2.58), we follow the asymptotic analysis carried out in [65]. This analysis must be somewhat subtle because the limit $q^{2} \rightarrow 0$ is singular for the Equation (2.58). Therefore, as in [65], we use the result of [132] to expand the probability density $\varrho_{0}(x)$ in Equation (2.65) in terms of the small ratio $q^{2} /(1+\bar{g})$ as

$$
\begin{aligned}
& \varrho_{0}(x) \sim \frac{m \tau}{x q}\left\{\exp \left(\frac{(1+\bar{g})\left[\eta^{2}\left(1+\bar{g}_{0}\right)-\eta^{2}(x)\right]}{2 q^{2}}\right)\right. \\
& \times\left[\sqrt{\frac{2}{1+\bar{g}}} D\left(\frac{\eta\left(1+\bar{g}_{0}\right)}{q} \sqrt{\frac{1+\bar{g}}{2}}\right)-\frac{q}{(1+\bar{g}) \eta\left(1+\bar{g}_{0}\right)}+\frac{q}{\bar{g}_{0}-\bar{g}}\right] \\
& \left.-\left[\sqrt{\frac{2}{1+\bar{g}}} D\left(\frac{\eta(x)}{q} \sqrt{\frac{1+\bar{g}}{2}}\right)-\frac{q}{(1+\bar{g}) \eta(x)}+\frac{q}{x-1-\bar{g}}\right]\right\} \text {. }
\end{aligned}
$$

where

$$
\eta(x)=\operatorname{sign}(x-1-\bar{g}) \sqrt{2\left(\frac{x}{1+\bar{g}}-1-\ln \frac{x}{1+\bar{g}}\right)},
$$

and $D(\cdot)$ stands for the Dawson integral

$$
D(z)=e^{-z^{2}} \int_{0}^{z} e^{y^{2}} d y .
$$

The two-term asymptotic expansion (2.68) is uniform in $x, \bar{g}$, and $q$.

In the small-fluctuation limit $q^{2} \ll \bar{g}$ and away from the mean-input value $\bar{g}=$ $\bar{g}_{0}$, which corresponds to the corner of the mean-field gain curve in Figure 2.1, the approximation (2.68) can be further simplified using the asymptotic relation

$$
D(z) \sim 1 / 2 z+O\left(1 / z^{3}\right)
$$

In particular, in the fluctuation- and mean-driven regimes, the approximation (2.68) simplifies to the point that we can obtain explicit expressions for the local portions of the gain curves there. We discuss these simplifications next. 
Fluctuation-Driven Regime: $\quad$ In this regime, $\bar{g}<\bar{g}_{0}\left(V_{S}<V_{T}\right.$, cf. Equation (2.18)). It is easy to analyze the function $\eta^{2}(x)$ using Equation (2.69) and conclude that it has a minimum at $x=1+\bar{g}<1+\bar{g}_{0}$. Therefore, using Equation (2.71), we find that, in Equation (2.68), the appropriate leading-order term in the expansion of the density $\varrho_{0}(x)$ in terms of the parameter $q^{2} /(1+\bar{g})$ is expressed as

$$
\varrho_{0}(x) \sim \frac{m \tau}{(1+\bar{g})\left(\bar{g}_{0}-\bar{g}\right)} \exp \left(\frac{(1+\bar{g}) \eta^{2}\left(1+\bar{g}_{0}\right)}{2 q^{2}}\right) \exp \left(-\frac{(x-1-\bar{g})^{2}}{2 q^{2}(1+\bar{g})}\right) .
$$

This formula has a relative error of $O\left(q^{2} /(1+\bar{g})\right)$ near $x=1+\bar{g}$, and is exponentially accurate everywhere else.
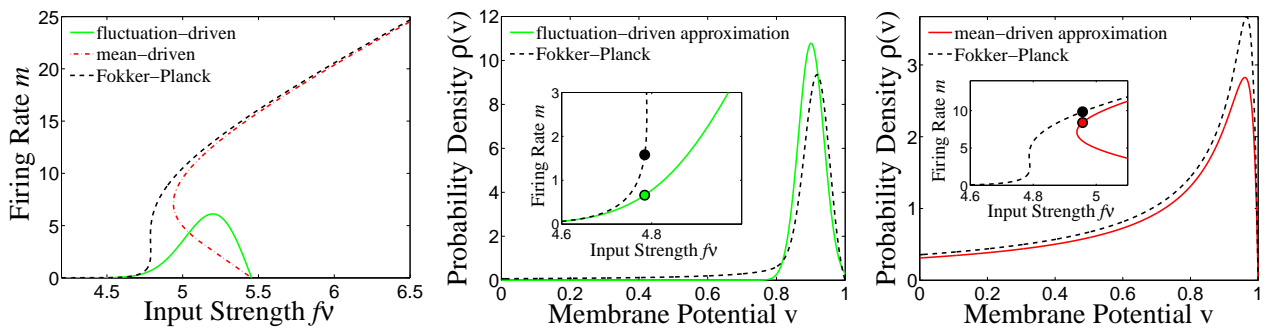

FIG. 2.6. Left: Comparison between a Fokker-Planck gain curve and its approximations in the fluctuation-driven and mean-driven regimes, given by Equations (2.75) and (2.17), respectively. Middle: Comparison between a Fokker-Planck voltage pdf and its Gaussian approximation (2.76) in the fluctuation-driven regime. Right: Comparison between a Fokker-Planck voltage pdf and its meanfield approximation with a boundary layer at $v=V_{T}$, given by Equation (2.78), in the mean-driven regime. [Reproduced with permission from Ref. [65], (Copyright 2009, by APS).]

Using the normalization condition (2.64), we integrate Equation (2.72) to find

$$
\frac{m \tau q}{\bar{g}_{0}-\bar{g}} \exp \left(\frac{(1+\bar{g}) \eta^{2}\left(1+\bar{g}_{0}\right)}{2 q^{2}}\right) \sqrt{\frac{\pi}{2(1+\bar{g})}}\left[1+\operatorname{erf}\left(\frac{\bar{g}}{\sqrt{2(1+\bar{g})} q}\right)\right] \sim 1,
$$

where $\operatorname{erf}(\cdot)$ denotes the error function $\operatorname{erf}(z)=(2 / \sqrt{\pi}) \int_{0}^{z} e^{-t^{2}} d t$, which implies that the firing rate $m$ is exponentially small in $q^{2} /(1+\bar{g})$, and so, asymptotically,

$$
\bar{g} \sim f \nu, \quad q^{2} \sim \frac{f^{2} \nu}{2 \tau} .
$$

This implies that the network dynamics in the fluctuation-driven regime are essentially feedforward.

Away from the external drive values $f \nu=0$ and $f \nu=\bar{g}_{0}$, Equations (2.73) and (2.74) imply the leading-order behavior for the firing rate to be

$$
m \sim\left(\bar{g}_{0}-f \nu\right) \sqrt{\frac{1+f \nu}{\pi \tau f^{2} \nu}} \exp \left\{\frac{2 \tau}{f^{2} \nu}\left[(1+f \nu)\left(1+\ln \frac{1+\bar{g}_{0}}{1+f \nu}\right)-1-\bar{g}_{0}\right]\right\},
$$

which, in turn, together with Equations (2.59), (2.72), and (2.74), gives for the voltage pdf, $\rho^{(v)}(v)$, the expression

$$
\rho^{(v)}(v) \sim \sqrt{\frac{\tau(1+f \nu)^{3}}{\pi f^{2} \nu}} \frac{1}{\varepsilon_{E}-\varepsilon_{r}} \exp \left[-\frac{\tau(1+f \nu)^{3}}{f^{2} \nu}\left(\frac{v-V_{S}}{\varepsilon_{E}-\varepsilon_{r}}\right)^{2}\right],
$$


where Equation (2.18) implies

$$
V_{S} \sim \frac{\varepsilon_{r}+f \nu \varepsilon_{E}}{1+f \nu}
$$

In the left and middle panels of Figure 2.6, we show a comparison between the gain curve computed from Equation (2.75) and that shown in the middle panel of Figure 2.5, which shows good agreement throughout the fluctuation-driven regime. This agreement also extends to the shapes of the respective voltage pdfs even close to the boundary of the fluctuation-driven regime, as displayed in the middle panel of Figure 2.6. In the limit of vanishing synaptic-input fluctuations, $f \rightarrow 0$ with $f \nu=\mathcal{O}(1)$, the Gaussian in Equation (2.76) becomes the delta function, $\rho^{(v)}(v) \rightarrow \delta\left(v-V_{S}\right)$, in agreement with Equation (2.19).

Mean-Driven Regime: $\quad$ In this regime, $\bar{g}>\bar{g}_{0}\left(V_{S}>V_{T}\right.$, cf. Equation (2.18)), and the function $\eta^{2}(x)$ (cf. Equation (2.69)) decreases monotonically for $1<x<1+\bar{g}_{0}$. Using Equation (2.71), we find that, in Equation (2.68), the leading-order term in the expansion of $\varrho_{0}(x)$ in terms of $q^{2} /(1+\bar{g})$ is thus given as

$$
\varrho_{0}(x) \sim \frac{m \tau}{x(1+\bar{g}-x)}\left\{1-\exp \left[-\frac{1}{q^{2}}\left(\frac{1+\bar{g}}{1+\bar{g}_{0}}-1\right)\left(1+\bar{g}_{0}-x\right)\right]\right\},
$$

corresponding to the voltage pdf

$$
\rho^{(v)}(v) \sim \frac{m \tau}{(1+\bar{g})\left(V_{S}-v\right)}\left[1-\exp \left(-\frac{\left(\bar{g}-\bar{g}_{0}\right)\left(V_{T}-v\right)}{q^{2}\left(\varepsilon_{E}-V_{T}\right)}\right)\right],
$$

which, in light of Equation (2.18), is the same as the voltage pdf in Equation (2.14), with the exception of an $\mathcal{O}\left(q^{2} /(1+\bar{g})\right)$-size boundary layer close to the threshold $v=V_{T}$. The difference stems from the boundary condition (2.48), which is dropped in the mean-driven limit. Normalization (2.16) implies that the leading-order behavior of the gain curves is again described by the mean-field parametrization (2.17).

In the left and right panels of Figure 2.6, we display a comparison between the mean-driven gain curve computed using Equation (2.17) and again the gain curve shown in the middle panel of Figure 2.5. One can observe good agreement throughout the mean-driven regime. The shapes of the respective voltage pdfs also agree very well even close to the boundary of the mean-driven regime, as shown in the right panel of Figure 2.6.

2.5.5. Validity of the Fokker-Planck description. To assess the validity of the approximate steady Fokker-Planck solutions, discussed in the previous two sections, we compare them with the results of direct numerical simulations of the I\&F system (2.1) [65]. (In fact, in our simulations, the conductances have both finite decay and rise times, so that the decaying exponential solutions of Equation (2.1b) are replaced by the alpha-curve-type functions $G(t)=$ $\theta(t)\left[\exp \left(-t / \sigma_{d}\right)-\exp \left(-t / \sigma_{r}\right)\right] /\left(\sigma_{d}-\sigma_{r}\right)$, were $\theta(t)$ is the Heaviside function, i.e., $\theta(t)=1$ for $t>0$ and 0 otherwise, and $\sigma_{d}$ stands for the $\sigma$ in Equation (2.1b). The chosen time-scales were $\sigma_{r}=0.1$ and $\sigma_{d}=0.2$ milliseconds.)

As shown in the left panel of Figure 2.7, the approximation of the gain curves improves with decreasing size of the synaptic-input fluctuations (i.e., the size of the external-spike strength $f$ ). Nevertheless, the approximation does not appear ever to be uniform, as the computed and theoretical gain curves clearly diverge away from one another for increasing external driving strength, $f \nu \gg \bar{g}_{0}$, and only converge 

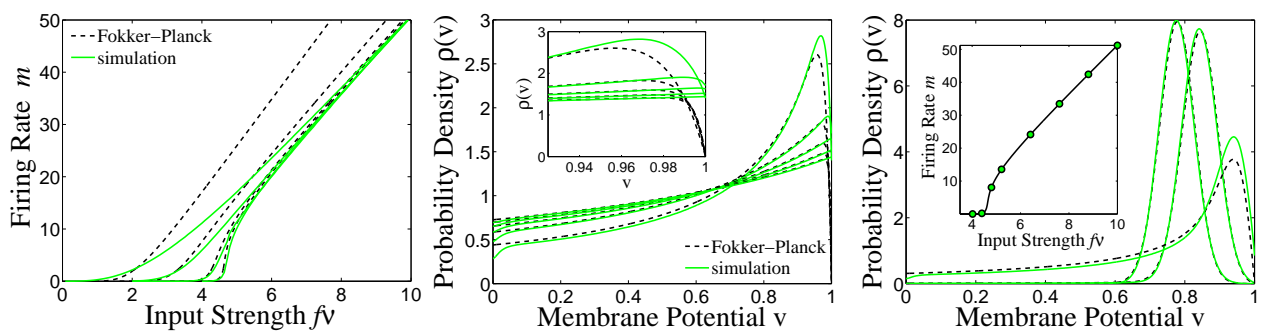

FIG. 2.7. Left: Comparison between Fokker-Planck gain curves and those computed via numerical simulations. The external spike strength $f$ decreases and network size $N$ increases from the left to the right curve, effectively decreasing the amount of synaptic-inputs fluctuations, going through the same values as in the right panel in Figure 2.1. Note the incorrect asymptotic slopes of the Fokker-Planck gain curves, which imply a non-uniform approximation. Middle and Right: Comparison between Fokker-Planck voltage pdfs and those computed via numerical simulations for a small amount of fluctuations in the fluctuation-and mean-driven regimes, respectively. Left Inset: The increasing heights of the locations along the gain curve correspond to the decreasing peak heights of the pdfs. Right Inset: The voltage pdfs obtained from simulations do not satisfy the boundary condition (2.48) due to finite rise and decay times of the conductances. [Reproduced with permission from Ref. [65], (Copyright 2009, by APS).]

towards one-another uniformly in finite intervals of $f \nu$. This discrepancy seems to be due to the failure of the diffusion approximation leading to the Fokker-Planck equation. We should remark that, since the kinetic equations do not rely on the diffusion approximation, we conjecture that the gain curves obtained using them should not suffer from the same deficiency. We have developed an asymptotic theory confirming this conjecture, which will be presented elsewhere.

The right two panels of Figure 2.7 show that, for small values of synaptic-input fluctuations, the computed and theoretical voltage pdfs agree very well, except in a boundary layer near the firing threshold $V_{T}$. This minor discrepancy arises due to the finite conductance time-scale $\sigma$ in the simulations, in particular, this scale being still relatively large as compared to the size of the synaptic-input fluctuations, which contradicts the assumption that $\sigma \rightarrow 0$ for which the boundary condition (2.48) of the Fokker-Planck description is valid [65].

We should remark that, to compute the gain curve corresponding to the smallest value of the synaptic-input fluctuations, we had to include random synaptic delays in the simulation, to break the tendency of the network to begin oscillating [65]. These delays do not alter the steady voltage pdfs or gain curves in our statistical description of the asynchronous dynamical regime of the network. The network oscillations tend to appear in the regime of small fluctuations and short excitatory conductance time-scale. In the limit of an infinitely fast conductance time-scale, these oscillations manifest themselves as firing cascades, during which the model neurons all fire at once at roughly periodic intervals $[80,81]$, and are a stochastic equivalent of the classical synchronization phenomenon described in [79]. Similar oscillations in the case of a finite excitatory conductance time-scale were studied in [53].

2.5.6. Bistability and neuronal orientation selectivity. The bistability of the firing rates, discussed in Sec 2.5.3, which can be observed in the mean-field gain curves (2.17) and in the small-fluctuation regime of the Fokker-Planck Equation (2.50), manifests itself as a hysteresis that can be observed in simulations. In particular, if we slowly ramp the external-driving strength $f \nu$ first up and then down through the 
bistability interval [130], we will make the network firing rate trace out first the bottom and then the top stable branches of the gain curve, and jump to the remaining stable branch of the gain curve close to each of the saddle-node bifurcation points at which one stable branch disappears. An example hysteresis loop for the small-fluctuation regime is presented in the left panel of Figure 2.8 .
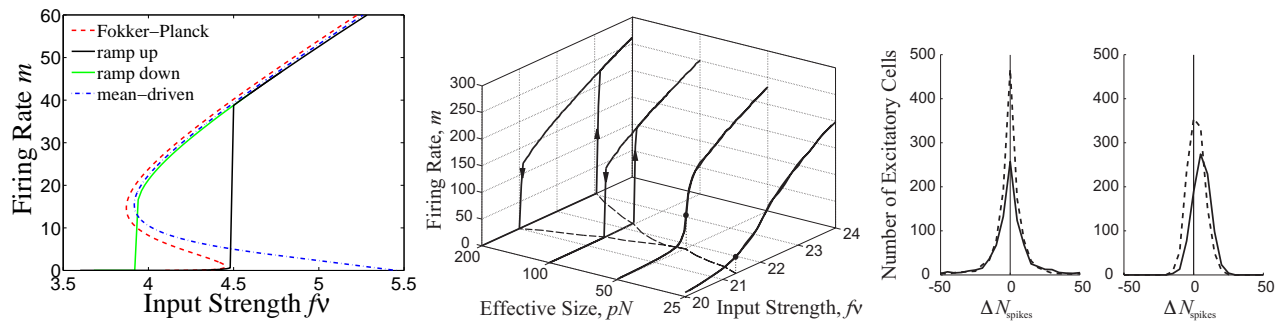

FIG. 2.8. Left: Bistability in Fokker-Planck and mean-field gain curves, and hysteresis computed in numerical simulations by slowly ramping the external-drive strength, $f \nu$, up and down, for the IES $F$ network (2.1) of effective size $p N=500$. Middle: Bifurcation to hysteresis in a sparsely-connected network of excitatory and inhibitory, simple and complex cells. The amount of fluctuations is related to the sparsity of the network connections. Right: Simulation results for a large-scale computational model of the primary visual cortex show that neither simple (dashed line) nor complex (solid line) cells exhibit hysteresis for small effective network size, pN, i.e., in the fluctuation-driven regime, as depicted in the left panel. Complex cells exhibit hysteresis for large effective network size, pN, i.e., in the mean-driven regime, as depicted in the right panel. (Cf. the main text of Section 2.5.6 and [130] for a detailed explanation.) [Reproduced with permission from Ref. [65], (Copyright 2009, by APS).][Reproduced with permission from Ref. [130], (Copyright 2006, by National Academy of Sciences, USA).]

Identifying hysteretic behavior played a significant role in properly tuning a largescale computational model of orientation-selective neurons in the primary visual cortex $[100,101,130]$. For small amounts of synaptic-input fluctuations in this model, slowly ramping the stimulus contrast, and therefore the external driving strength, up and down produces hysteretic behavior in complex cells, i.e., those neurons which receive their synaptic input primarily or entirely from other network neurons. This behavior manifests itself as a positive statistical difference, $\Delta N_{\text {spikes }}$, between the numbers of neuronal spikes during the down-ramping and up-ramping of the stimulus contrast. It is less pronounced for simple cells, i.e., those neurons which receive their synaptic input primarily or entirely from the external drive, and disappears when the amount of fluctuations increases. This amount is determined by the effective number of synaptic connections per neuron, which in turn is determined by the degree of network sparsity, in other words, the probability of a synaptic connection existing between a pair of neurons. Sparser networks with fewer synaptic connections experience more input fluctuations, and so operate in the fluctuation-driven regime, while those with more synaptic connections operate in the mean-driven regime.

A model of intermediate complexity clearly exhibits a similar type of hysteretic behavior $[100,101,130]$. In this model, $50 \%$ of the neurons are simple cells which receive external Poisson spike-train drive, all with identical rate $\nu$ and spike strength $f$. The other $50 \%$ of the neurons are complex cells, which only receive synaptic input from other network neurons; the coupling coefficients are chosen so that the average amount of excitation each neuron receives is approximately the same. All the neurons receive strong inhibition from the inhibitory neurons in the network. The network couplings are sparse, with $p$ denoting the probability of the existence of a connec- 
tion between a pair of neurons, and thus $p N$ giving the effective network size, where $N$ is the number of neurons in the network. The size $p N$ controls the amount of synaptic-input fluctuations in the network; the larger the size, the fewer fluctuations. The bifurcation diagram in the middle panel of Figure 2.8 shows hysteretic behavior similar to that exhibited by an all-excitatory network depicted in the left panel for sufficiently few synaptic-input fluctuations. This diagram also presents a bifurcation scenario identical to that exhibited by the steady solutions of the Fokker-Planck Equation (2.50), depicted in Figure 2.5, i.e., the gradual removal of bistability when the amount of synaptic-input fluctuations increases.

Experimentally, it was shown that cortical neurons' degree of orientation selectivity is independent of the stimulus contrast, and that this prompts the network to operate in a high-gain regime, as close to the transition to bistability as possible, but that no bistable behavior of simple or complex cells has been observed in the input layer of the primary visual cortex $[3,5,46,47,107,110,111,119]$. The constraints laid out by this fluctuation-controlled criticality scenario allows us to tune the large-scale model network so that it operates in the regime just below the critical transition point to bistability $[100,101,130]$, whose existence and properties are well understood through our kinetic-theoretic coarse-graining approach. In other words, the simple steady solutions of the Fokker-Planck Equation (2.50) appear sufficient to capture the mechanism underlying the hysteresis present in the large-scale cortical model and pinpoint where its operating regime should be to best reproduce the experimental facts.

\section{Network connectivity fluctuations}

In the true networks of neurons in the brain, the neurons are not coupled uniformly in an all-to-all fashion. Their connectivity architecture instead tends to be sparse $[9$, $73-76,118,133,134]$ and heterogeneous, with the numbers of synapses on any given neuron and the existence of a synaptic connection between any given pair of neurons fluctuating widely, yet with well identifiable statistical properties giving rise to both regular and disordered feature-preference maps on the scales spanning from a few tenths of a millimeter to several millimeters $[11,12,16,39,44,60,72,103,116,129$, 138, 142]. To best isolate the effects of such network-connectivity fluctuations, we study them separately from the synaptic-input fluctuations and therefore within the framework of the mean-field model.

In this section, we first discuss how to generalize the description of I\&F networks from all-to-all coupled, as in Equation (2.1), to heterogeneous coupling architecture, and follow by deriving the corresponding generalizations of the Boltzmann Equation (2.3) and the mean-field system (2.10). We then use a high-conductance version of the mean-field system to derive explicit expressions describing the neuronal firing rates deep in the mean-drive regime, where the synaptic-input fluctuations can be ignored, and study how the statistics of these firing rates depend on the underlying complex network topology.

3.1. Heterogeneously coupled integrate-and-fire networks. The I\&F system (2.1) can easily be generalized to networks with complex architecture. In such networks, the uniform coupling of Equation (2.1) may be replaced by pairwise coupling coefficients $a_{i j}$ determined by the network topology, and the index $i$ typically does not merely count neurons, but is also used to label some of their properties, such as the number of their pre- and postsynaptic connections or their cortical location. 
The resulting system becomes

$$
\begin{aligned}
\tau \frac{d V_{i}}{d t} & =-\left(V_{i}-\varepsilon_{r}\right)-G_{i}\left(V_{i}-\varepsilon_{E}\right) \\
\sigma \frac{d G_{i}}{d t} & =-G_{i}+f \sum_{\mu} \delta\left(t-t_{\mu}^{i}\right)+\frac{S}{\phi(N)} \sum_{j \neq i} \sum_{\mu} a_{i j} p_{i j \mu} \delta\left(t-t_{j \mu}\right),
\end{aligned}
$$

where $\phi(N)$ is some increasing function of the network size $N$ which is related to the effective network connectivity and ensures finite firing rates in the large-network limit, $N \rightarrow \infty$. Determining the form of the function $\phi(N)$ is typically not trivial, and is part of the problem of determining a proper statistical description of the network (3.1). We still assume that the Poisson rate of the external-drive spike-train arriving at each network neuron is the same.

3.1.1. Boltzmann and advection-diffusion equations for a complex network. From now on, we discuss model neuronal networks with complex connectivity topology but identical coupling strengths between pairs of neurons, which corresponds to the assumption that two neurons are either connected by one synapse or none. In this case, each of the coupling coefficients $a_{i j}$ in Equation (3.1) equals either 1 or 0 .

An important quantity one has to take into account in networks with complex connectivity topology, and thus include its dependence in the index $i$ in Equation (3.1), is the incoming connectivity degree of the neurons in the network, i.e., the number of neurons which synapse onto a given neuron. Since the entire network spike train arriving at a neuron is the sum of the spike trains arriving from each of its presynaptic neurons, one should expect this neuron's incoming degree to be closely connected to its firing rate. Therefore, we partition the network into groups of neurons with like incoming connectivity degrees, and study the coupled dynamics of the neurons that belong to these groups. Since, typically, the incoming degree, $k$, of neurons is only known statistically, we study its probability distribution, $P_{\text {in }}(k)$. We refer to neurons with incoming degree $k$ as $k$-neurons. We also need to include information concerning the distribution of the types of synapses present in the network, which is described by the probability $T(n, k)$ of finding a synapse originating at an $n$-neuron and terminating at a $k$-neuron. The function $T(n, k)$ is alternatively known as the degree-correlation function $[40,84]$.

A simple counting argument gives the relationship between the distributions $P_{\text {in }}(k)$ and $T(n, k)$ as

$$
\sum_{n=0}^{N-1} T(n, k)=\frac{k P_{\text {in }}(k)}{\mu},
$$

where

$$
\mu=\sum_{n=0}^{N-1} k P_{\text {in }}(k)
$$

stands for the mean incoming degree of the neurons in the network. Likewise, we find the conditional probability $P(n \mid k)$ of a synapse to originate at an $n$-neuron if we 
know that it terminates at a $k$-neuron to be

$$
P(n \mid k)= \begin{cases}\frac{\mu T(n, k)}{k P_{\mathrm{in}}(k)}, & k \geq 1, \\ 0, & k=0 .\end{cases}
$$

In view of this formula, we will use the convention $T(n, k) / k P_{\text {in }}(k) \equiv 0$ for $k=0$ in all our further derivations.

A slight generalization of the derivation presented in Section 2.2 yields the system of Boltzmann differential-difference equations governing the evolution of the joint voltage-conductance probability density $\rho_{k}(v, g, t)$ of a typical $k$-neuron. This system, analogous to Equation (2.3), reads

$$
\begin{aligned}
\partial_{t} \rho_{k}=\partial_{v} & \left\{\left[\left(\frac{v-V_{r}}{\tau}\right)+g\left(\frac{v-V_{E}}{\tau}\right)\right] \rho_{k}\right\}+\partial_{g}\left(\frac{g}{\sigma} \rho_{k}\right) \\
& +\nu(t)\left[\rho_{k}\left(v, g-\frac{f}{\sigma}, t\right)-\rho_{k}(v, g, t)\right] \\
& +p k \mu_{k}(t)\left[\rho_{k}\left(v, g-\frac{\bar{S}}{p \sigma \phi(N)}, t\right)-\rho_{k}(v, g, t)\right],
\end{aligned}
$$

where

$$
\mu_{k}(t)=\sum_{n=0}^{N-1} P(n \mid k) m_{n}=\frac{\mu}{k P_{\text {in }}(k)} \sum_{n=0}^{N-1} T(n, k) m_{n}
$$

is the average input received by a $k$-neuron from other neurons, $P(n \mid k)$ is the conditional probability defined in Equation (3.4), $\bar{S}=p S$, with $p$ being the synaptic release probability, and $\phi(N)$ the scaling factor used in Equation (3.1).

The voltage and conductance probability fluxes $J_{k, V}(v, g, t)$ and $J_{k, G}(v, g, t)$, defined by analogy with Equation (2.5), allow us to rewrite the system (3.5) in the conservation form

$$
\partial_{t} \rho_{k}(v, g, t)+\partial_{v} J_{k, V}(v, g, t)+\partial_{g} J_{k, G}(v, g, t)=0 .
$$

The boundary conditions analogous to Equation (2.6) are

$$
J_{k, V}\left(V_{T}, g, t\right)=J_{k, V}\left(\varepsilon_{r}, g, t\right),
$$

for $0 \leq g<\infty$,

$$
\rho_{k}(v, g<0, t) \equiv 0,
$$

and

$$
\rho_{k}(v, g \rightarrow \infty, t) \rightarrow 0,
$$

sufficiently rapidly, together with all their derivatives, the latter two conditions valid for $\varepsilon_{r} \leq v<V_{T}$. The firing rate condition (2.6d) is replaced by the set of conditions

$$
\begin{aligned}
m_{k}(t) & =\int_{0}^{\infty} J_{k, V}\left(V_{T}, g, t\right) d g \\
& =-\int_{0}^{\infty}\left[\left(\frac{V_{T}-\varepsilon_{r}}{\tau}\right)+g\left(\frac{V_{T}-\varepsilon_{E}}{\tau}\right)\right] \rho_{k}\left(V_{T}, g, t\right) d g .
\end{aligned}
$$


Equations (3.5), together with the boundary conditions (3.8a), (3.8b), and (3.8c), and the nonlinear self-consistency conditions (3.8d), render a complete Boltzmanntype kinetic description of the neuronal network (3.1) as an infinite system of partial differential equations for the densities $\rho_{k}(v, g, t)$. These equations are all coupled nonlinearly because the multiplicative coefficients $\mu_{k}(t)$, defined in Equation (3.6), couple all the firing rates obtained from the boundary terms in Equation (3.8d).

If we assume the jumps a neuron's conductance makes upon receiving a spike to be small, we can Taylor-expand the corresponding jump terms, described on the second and third lines of Equation (3.5), to second order, and derive the advection-diffusion equation

$$
\partial_{t} \rho_{k}=\partial_{v}\left\{\left[\left(\frac{v-V_{r}}{\tau}\right)+g\left(\frac{v-V_{E}}{\tau}\right)\right] \rho_{k}\right\}+\partial_{g}\left[\frac{1}{\sigma}\left(g-\bar{g}_{k}(t)\right) \rho_{k}+\frac{\sigma_{k}^{2}(t)}{\sigma} \partial_{g} \rho_{k}\right],
$$

where

$$
\bar{g}_{k}(t)=f \nu(t)+\frac{\bar{S}}{\phi(N)} k \mu_{k}(t)
$$

is the mean synaptic input to a $k$-neuron, and

$$
\sigma_{k}^{2}(t)=\frac{1}{2 \sigma}\left[f^{2} \nu(t)+\frac{\bar{S}^{2}}{p[\phi(N)]^{2}} k \mu_{k}(t)\right]
$$

represents this neuron's variance of synaptic input fluctuations.

3.1.2. Mean-field Approximation. To address the effects of complex network architecture on the neuronal firing rates, it suffices to begin with considering the fluctuationless operating regime of the network (3.1), in which the synaptic-input fluctuations of any neuron become negligible as compared to its mean synaptic input. In other words, in a complex network, we consider the case in which $\sigma_{k}^{2}(t) / \bar{g}_{k}(t) \rightarrow 0$ for the quantities in Equation (3.10) and all neuronal incoming degrees $k$. As in Section 2.3, after assuming the conductance and voltage pdfs of all $k$-neurons to be statistically independent [31],

$$
\rho_{k}(v, g, t)=\rho_{k}^{(v)}(v, t) \rho_{k}^{(g)}(g, t)
$$

we find the coupled system of mean-field equations, analogous to Equation (2.10), expressed as

$$
\begin{aligned}
\partial_{t} \rho_{k}^{(v)}(v, t) & =\partial_{v}\left\{\left[\left(\frac{v-V_{r}}{\tau}\right)+\langle g\rangle_{k}(t)\left(\frac{v-V_{E}}{\tau}\right)\right] \rho^{(v)}(v, t)\right\}, \\
\frac{d}{d t}\langle g\rangle_{k}(t) & =-\frac{1}{\sigma}\left[\langle g\rangle_{k}(t)-\bar{g}_{k}(t)\right],
\end{aligned}
$$

where

$$
\langle g\rangle_{k}(t)=\int_{0}^{\infty} g \rho_{k}^{(g)}(g, t) d g
$$

is the expected conductance value of a $k$-neuron, and $\bar{g}_{k}(t)$ is the mean synaptic input to a $k$-neuron, defined above in Equation (3.10a). The analog of the boundary 
conditions (2.13) is the set of conditions

$$
\begin{aligned}
m_{k}(t) & =\langle g\rangle_{k}(t)\left(\frac{\varepsilon_{E}-\varepsilon_{r}}{\tau}\right) \rho_{k}^{(v)}\left(\varepsilon_{r}, t\right) \\
& =-\left[\left(\frac{V_{T}-\varepsilon_{r}}{\tau}\right)+\langle g\rangle_{k}(t)\left(\frac{V_{T}-\varepsilon_{E}}{\tau}\right)\right] \rho_{k}^{(v)}\left(V_{T}, t\right) .
\end{aligned}
$$

For time-independent external drive, Equation $(3.12 \mathrm{~b})$ relaxes to the solution $\langle g\rangle_{k}=\bar{g}_{k}$. After one integration, Equation (3.12a) then gives the expression for the voltage pdf of $k$-neurons as

$$
\rho_{k}^{(v)}(v)=-\frac{\tau m_{k}}{\left(v-V_{r}\right)+\bar{g}_{k}\left(v-V_{E}\right)} .
$$

The normalization condition (2.16) for the voltage $\operatorname{pdf} \rho_{k}^{(v)}(v)$ finally yields the implicit equation for the firing rate $m_{k}$,

$$
\tau m_{k}=\frac{1+\bar{g}_{k}}{\log \left|\frac{\bar{g}_{k}\left(\varepsilon_{r}-\varepsilon_{E}\right)}{V_{T}-\varepsilon_{r}+\bar{g}_{k}\left(V_{T}-\varepsilon_{E}\right)}\right|} .
$$

Here the mean synaptic input $\bar{g}_{k}$ in Equation (3.10a) and the firing rate $m_{k}$ of the $k$-neurons are related via Equation (3.6), which also couples all the firing rates $m_{k}$.

3.1.3. High-conductance limit in the mean-driven regime. Since we are interested in isolating the effects of complex network topology on the firing rates of different network neurons, and want to eliminate any interference from synaptic-input fluctuations, we focus our study on the firing-rate behavior deep in the mean-driven regime. In particular, we consider the high-conductance limit in which the average synaptic input of the $k$-neurons, $\bar{g}_{k}$, is strong, $\bar{g}_{k} \gg \bar{g}_{0}$, where $\bar{g}_{0}$ is defined as in Equation (2.15). We can then Taylor-expand the right-hand side of Equation (3.15) in terms of the small quantity $1 / \bar{g}_{k}$, and keep only the terms of $\mathcal{O}(1)$ or larger, to obtain the following linear asymptotic approximation of the original system (3.15):

$$
\begin{aligned}
\tau m_{k} \ln \left(1+\bar{g}_{0}\right) & =1+\bar{g}_{k}-\frac{\bar{g}_{0}}{\ln \left(1+\bar{g}_{0}\right)} \\
& =f \nu+1-\frac{\bar{g}_{0}}{\ln \left(1+\bar{g}_{0}\right)}+\frac{\mu \bar{S}}{p \phi(N) P_{i n}(k)} \sum_{n=0}^{N-1} T(n, k) m_{n} .
\end{aligned}
$$

System (3.16) is the complex-network analog of the asymptote (2.20).

3.2. Dependence of the firing rate on the network architecture. For three representative networks with connectivity architectures of increasing complexity, we now use the high-conductance limit of the mean-field Equation (3.16) to derive explicit asymptotic expressions for the firing rates $m_{k}$ of the $k$-neurons deep in the mean-driven regime. We use these expressions to study how the rates $m_{k}$ depend on the underlying features of the network topology. We also study the same dependence for the mean firing rate,

$$
\bar{m}=\sum_{k=0}^{N-1} m_{k} P_{\text {in }}(k),
$$

of all the neurons in the network. 
3.2.1. Uncorrelated network. In an uncorrelated network, the conditional probability $P(n \mid k)$ of a synapse to originate at an $n$-neuron, provided we know that it terminates at a $k$-neuron, must be independent of the index $k$ of the postsynaptic neuron. Formula (3.4) and counting of synapses then immediately imply that, for such networks, the edge-type distribution can be expressed in terms of the corresponding incoming-degree distributions as a product in the form

$$
T(n, k)=k P_{\text {in }}(k) n P_{\text {in }}(n) / \mu^{2} .
$$

Uncorrelated networks include random networks of Erdös-Rényi type [42,43,51], as well as random scale-free networks $[35,40]$. Recall that scale-free networks are defined as those with a power-law asymptotic behavior of the incoming degree distribution, $P_{\text {in }}(n) \sim n^{-\gamma}$. Particularly interesting are scale-free networks for which $2<\gamma \leq 3$. In such networks, the mean, $\mu$, of the distribution $P_{\text {in }}(n)$ remains finite, while its second moment,

$$
\left\langle n^{2}\right\rangle_{N}=\sum_{n=0}^{N-1} n^{2} P_{\text {in }}(n)
$$

as computed from Equation (3.17), diverges as the size of the network, $N$, increases.

A guess that the firing rate $m_{k}$ of the $k$-neurons is a linear function of the neuronal incoming degree $k$ when Equation (3.18) holds leads to the solution of Equation (3.16) given by

$$
m_{k}=\frac{1}{\ln \left(1+\bar{g}_{0}\right)}\left(f \nu+1-\frac{\bar{g}_{0}}{\ln \left(1+\bar{g}_{0}\right)}\right)\left(1+\frac{\lambda k}{1-\lambda\left\langle n^{2}\right\rangle_{N} / \mu}\right)
$$

where

$$
\lambda=\frac{\bar{S}}{\tau p \phi(N) \ln \left(1+\bar{g}_{0}\right)}
$$

is the rescaled coupling coefficient. As mentioned above, this solution approximates the firing rate $m_{k}$ asymptotically deep in the mean-driven regime [113].

Equation (3.20) implies linear dependence of the firing rate $m_{k}$ on both the driving strength, $f \nu$, and the incoming degree of the neuron, $k$. Note that all the $m-f \nu$ gain curves become singular at $\lambda=\mu /\left\langle n^{2}\right\rangle_{N}$, when their slopes become infinite. In the limit of large network size $N$, the scaling factor $\phi(N)$ in the rescaled coupling coeffcient $\lambda$ must equal the ratio of the first and second moments, $\mu /\left\langle n^{2}\right\rangle_{N}$, of the node-degree distribution, $P_{\text {in }}(n)$, for there to be a finite interval of the coupling coefficients $\bar{S}$ for which the firing rates $m_{k}$ are finite. Under this scaling, the network-averaged firing rate

$$
\bar{m}=\frac{1}{\ln \left(1+\bar{g}_{0}\right)}\left(f \nu+1-\frac{\bar{g}_{0}}{\ln \left(1+\bar{g}_{0}\right)}\right)\left(1+\frac{\lambda \mu}{1-\lambda\left\langle n^{2}\right\rangle_{N} / \mu}\right)
$$

also remains finite as $N \rightarrow \infty$ because $\left\langle n^{2}\right\rangle_{N}>\mu^{2}$.

3.2.2. Unidirectional scale-free network. In the network we discuss in this section, the neurons have their incoming degrees distributed according to a scalefree law, i.e., the distribution has power-law tails, while each neuron only sends spikes to one postsynaptic neuron $[113,114]$. The network grows in stages, beginning with 
one synaptic connection between a pair of neurons. At every stage, a single new presynaptic neuron is connected to a specific existing postsynaptic neuron in the network. The probability for the new neuron to be connected to an old network neuron is proportional to the old neuron's incoming degree [66].

The incoming-degree and edge-type distributions of the network neurons are expressed as

$$
P_{\text {in }}(k)=\frac{4}{(k+1)(k+2)(k+3)},
$$

and

$$
T(n, k)=\frac{4 k}{(n+1)(n+k+2)(n+k+3)(n+k+4)}\left[\frac{1}{n+2}+\frac{3}{n+k+1}\right],
$$

respectively [66,114]. Note that the distribution (3.23) behaves as a power law when the neuronal incoming degree $k$ is large. One can easily show that the mean incoming degree satisfies $\mu=1$ and that the incoming-degree variance satisfies $\left\langle n^{2}\right\rangle_{N} \sim \ln N$.
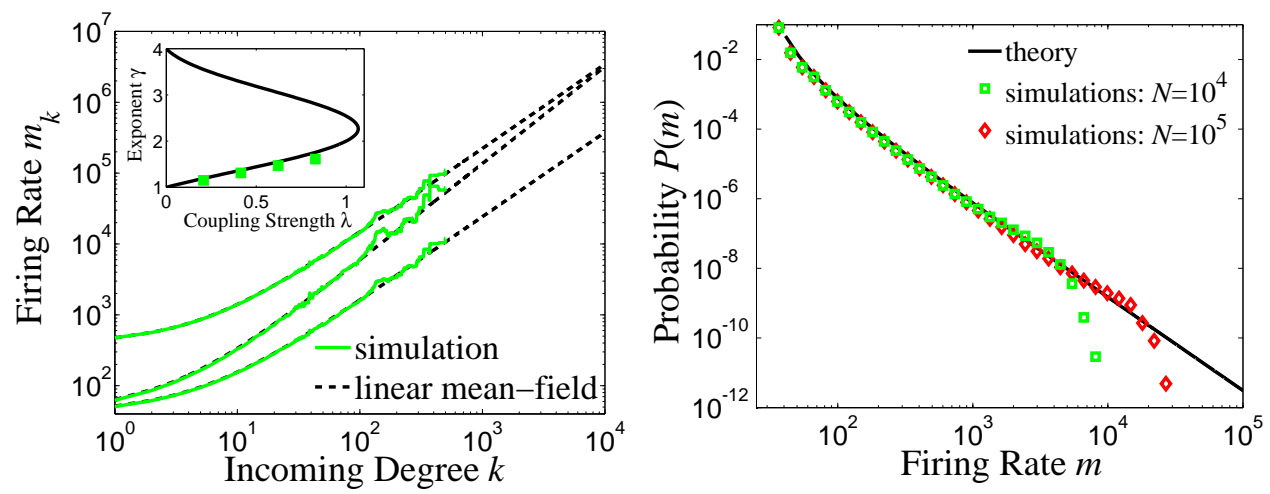

FIG. 3.1. Unidirectional scale-free network discussed in Section 3.2.2. Left: Power-law asymptotic behavior of the firing rate $m_{k}$ and its dependence on the driving strength $f \nu$ and coupling strength $\lambda$. The two parallel curves share the same value of $\lambda$. The middle and bottom curves share the same value of $f \nu$. Inset: The $\gamma$ versus $\lambda$ dependence as expressed by Equation (3.26) (black line) and deduced from full numerical simulations (gray squares, green online). Right: Two histograms of firing rates, computed numerically from network ensembles (squares and diamonds), and the firingrate distribution $P(m)$ predicted using Equation (3.34) below (black solid line). [Reproduced with permission from Ref. [114], (Copyright 2009, by Europhysics Letters Association).]

For large neuronal incoming degree, $k \gg 1$, and infinite size of the network, $N \rightarrow$ $\infty$, we find that the firing rate $m_{k}$ depends on the neuronal incoming degree $k$ as a power-law. Assuming the form of the solution of Equation (3.16) as

$$
m_{k} \sim B k^{\gamma}, \quad k \gg 1
$$

replacing summation with integration, evaluating an integral using residues, expanding in a series at large values of $k$, and collecting the leading-order terms [113], we find that the exponent $\gamma$ and the network coupling coefficient $\lambda$ in Equation (3.21) are related via the equation

$$
\lambda=-\frac{2 \sin (\pi \gamma)}{\pi \gamma(\gamma-2)(\gamma-3)}
$$


For both sides of Equation (3.16) to remain finite, the exponent $\gamma$ must satisfy the inequality $\gamma<4$, and because the network coupling $\lambda$ is nonnegative, $\gamma$ must satisfy $\gamma>1$. These two requirements leave us with two possible branches of the $\gamma$ versus $\lambda$ dependence as expressed by Equation (3.26), as shown in Figure 3.1. Numerical simulations of the corresponding I\&F network (3.1) indicate that the lower branch is stable $[113,114]$. Note that the scaling factor $\phi(N)$ in the coupling constant $\lambda$ can be of $\mathcal{O}(1)$, and the network will still maintain finite firing rates in the large-size limit, $N \rightarrow \infty$.

The mean firing rate, $\bar{m}$, can be found exactly using Equation (3.17) and the fact that every neuron's outgoing node-degree, i.e., the number of synapses originating at this neuron, equals 1 by construction. The probability $\sum_{k=0}^{N-1} T(n, k)$ of finding a synapse originating at an $n$-neuron is thus equal to the probability $P_{\text {in }}(n)$ of finding an $n$-neuron, and so using Equations (3.16) and (3.17) we can derive that

$$
\bar{m}=\frac{1}{\ln \left(1+\bar{g}_{0}\right)}\left(f \nu+1-\frac{\bar{g}_{0}}{\ln \left(1+\bar{g}_{0}\right)}\right) \frac{1}{1-\mu \lambda} .
$$

Note that the mean network firing rate $\bar{m}$ develops a singularity at $\lambda=1$, while the individual firing rates $m_{k}$ remain bounded.

3.2.3. Scale-free network. We finally derive the asymptotic firing rates for a degree-correlated neuronal network with scale-free distributions of both incoming and outgoing degrees [113]. Since neuronal networks are directed, we construct this scalefree network in two steps: we first construct the corresponding undirected network following the algorithm described in [61], and then randomly assign a direction to each of its undirected edges. The undirected network of [61] grows in stages: its first stage is an all-to-all connected network consisting of $\ell$ nodes, which are said to be active. At each subsequent stage of the network growth, a new active node is first attached to every active node via an undirected edge, and then an active node is deactivated with the probability $\sim 1 / n$, where $n$ is its current total degree, i.e., the number of all undirected edges emanating from it. A direction is assigned randomly to every edge, with probability $1 / 2$.

For the resulting directed network, if the initial number of active nodes, $\ell$, is large, $\ell \gg 1$, the incoming-degree and edge-type distributions are well-approximated by the expressions

$$
\begin{gathered}
P_{\text {in }}(k)=\frac{\ell^{2}}{2 k^{3}}, \\
T(n, k)=\frac{P_{\text {in }}(k) P_{\text {in }}(n)}{\ell}(n+k-\ell)
\end{gathered}
$$

for the incoming-degree values of $k, n \geq \ell / 2$, and vanish rapidly for $k, n<\ell / 2$, so that we approximate them as vanishing exactly there [113]. The incoming-degree distribution of this network is obviously scale-free. Up to terms that decay with growing network size $N \gg 1$, this network's first and second incoming-degree moments are

$$
\mu=\ell, \quad\left\langle n^{2}\right\rangle_{N}=\frac{\ell^{2}}{2} \ln \frac{N}{\ell},
$$

the first of which is indeed bounded, and the second is logarithmically increasing with network size $N$. 

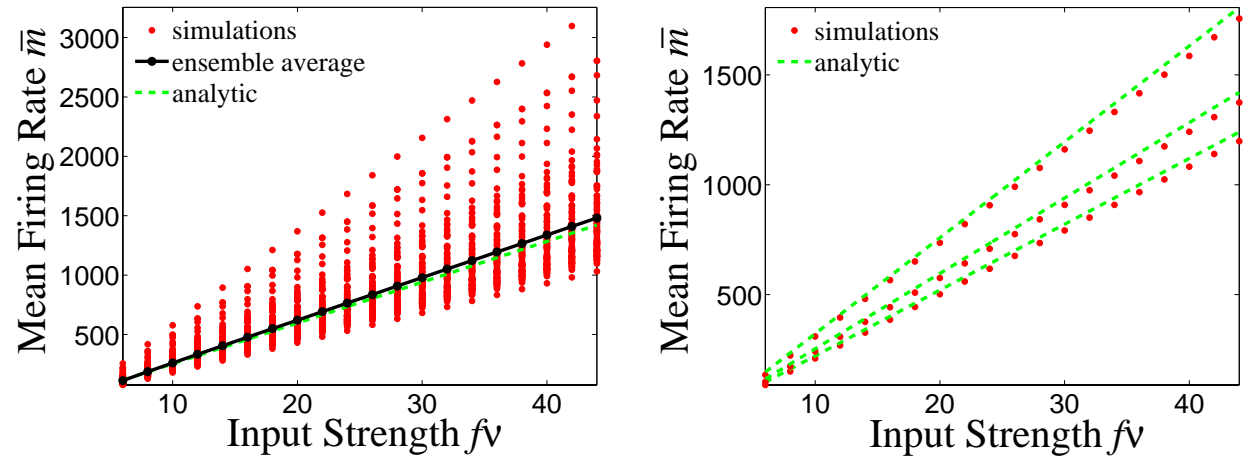

FIG. 3.2. Gain curves for the scale-free network discussed in Section 3.2.3. Left: Gain curve obtained from Equation (3.32) describes the dependence of the mean network firing rate $\bar{m}$ on the external-drive strength $f \nu$ on average for an ensemble of networks. Right: Equation (3.31) with the variance of the neuronal incoming-degree distribution replaced by the variance of the neuronal incoming-degrees in a given network realization describes the dependence of the mean network firing rate $\bar{m}$ on the external-drive strength $f \nu$ in that realization.

We again guess the solution for the average firing rate, $m_{k}$, of a $k$-neuron to be linear in its incoming degree, $k$, and find

$$
m_{k}=\frac{1}{\ln \left(1+\bar{g}_{0}\right)}\left(f \nu+1-\frac{\bar{g}_{0}}{\ln \left(1+\bar{g}_{0}\right)}\right)\left(1+\frac{\lambda k+\lambda^{2} \sigma_{N}^{2}}{1-\lambda \mu-\lambda^{2} \sigma_{N}^{2}}\right) .
$$

Equation (3.31) shows that the slope of the gain curve is linear in both the driving strength, $f \nu$, and the incoming-degree of the neurons, $k$, and becomes infinite at the coupling value $\lambda=\left(\mu+\sqrt{\mu^{2}+4 \sigma_{N}^{2}}\right) / 2$ for all values of $k$ simultaneously, exactly as for the uncorrelated network in Section 3.2.1. The scaling factor $\phi(N)$ in the coupling parameter $\lambda$ in Equation (3.21) must be chosen of $O\left(1 /\left\langle n^{2}\right\rangle_{N}^{1 / 2}\right)$ in order for an interval of parameters $\bar{S}$ to exist for which there is a stable steady state in the largenetwork limit, $N \rightarrow \infty$. Averaging Equation (3.31) over the neuronal incoming degree $k$ using Equation (3.17), we find the mean network firing rate

$$
\bar{m}=\frac{1}{\ln \left(1+\bar{g}_{0}\right)}\left(f \nu+1-\frac{\bar{g}_{0}}{\ln \left(1+\bar{g}_{0}\right)}\right) \frac{1}{1-\lambda \mu-\lambda^{2} \sigma_{N}^{2}},
$$

which remains bounded for the same values of the coupling parameter $\bar{S}$ as the individual firing rates $m_{k}$ in Equation (3.31) as the network size increases, $N \rightarrow \infty$.

Direct numerical Monte-Carlo simulations of Equation (3.1) confirm the validity of the mean-firing-rate formula in Equation (3.32) on average, for an ensemble of many networks. They also show that Equation (3.32) describes the mean gain curve of an individual network realization, provided the node-degree variance $\sigma_{N}^{2}$ of the distribution $P_{\text {in }}(n)$ is replaced by the value of the node-degree variance of this particular realization. Likewise, Equation (3.31) accurately describes the average firing rate, $m_{k}$, of the $k$-neurons in an ensemble of many networks. Moreover, Equation (3.31) also provides an accurate description of the average firing rate, $m_{k}$, of the $k$-neurons in a specific network realization, provided the incoming-degree variance $\sigma_{N}^{2}$ of $P_{\text {in }}(n)$ is again replaced by the realization variance. 
3.2.4. Firing-rate distribution. To find the probability distribution of the neuronal firing rates, we use the chain rule to find

$$
P(m)=P_{\text {in }}(k) \frac{d k}{d m},
$$

where $P_{\text {in }}(k)$ is the corresponding neuronal incoming-degree distribution. The neuronal incoming-degree, $k$, in Equation (3.33) must be expressed in terms of the firing rate by inverting the expression $m=m_{k}$.

For the unidirectional scale-free network of Section 3.2.2, we use Equations (3.23) and (3.33) to find the firing-rate distribution tail,

$$
P(m) \propto m^{-2 / \gamma-1},
$$

valid for high firing-rates. Likewise, Equations (3.31) and (3.33) imply that the scalefree distribution of Section 3.2.3 asymptotically gives rise to the firing-rate distribution tail

$$
P(m) \sim\left(f \nu+1-\frac{\bar{g}_{0}}{\ln \left(1+\bar{g}_{0}\right)}\right)^{2} \frac{\ell^{2} \lambda^{2}}{2\left[\ln \left(1+\bar{g}_{0}\right)\right]^{2}\left(1-\lambda \mu-\lambda \sigma_{N}^{2}\right)^{2} m^{3}},
$$

with $\mu$ and $\sigma_{N}^{2}$ as in Equation (3.30), and again valid for high firing-rates. Both distribution tails in Equations (3.34) and (3.35) are power laws.

3.3. Spatially extended networks. To describe spatially extended networks, we generalize our kinetic theory to cover Equation (3.1) by including interactions among the appropriate coarse-grained tissue patches created by a spatial coarsegraining procedure. When the coupling coefficients $a_{i j}$ only depend on the distance $|i-j|$, the coupling among such coarse-grained patches is accomplished by generalizing the mean synaptic input $\bar{g}(t)$ and synaptic-input fluctuations $\sigma_{g}^{2}(t)$ in Equations (2.11) and (2.23) as

$$
\begin{aligned}
\tilde{g}(t ; \mathbf{x}) & =f \nu(t ; \mathbf{x})+\bar{S} \int a\left(\mathbf{x}-\mathbf{x}^{\prime}\right) m\left(t ; \mathbf{x}^{\prime}\right) d \mathbf{x}^{\prime} \\
\sigma_{g}^{2}(t ; \mathbf{x}) & =\frac{1}{2 \sigma}\left[f^{2} \nu(t ; \mathbf{x})+\frac{\bar{S}^{2}}{p N} \int a^{2}\left(\mathbf{x}-\mathbf{x}^{\prime}\right) m\left(t ; \mathbf{x}^{\prime}\right) d \mathbf{x}^{\prime}\right],
\end{aligned}
$$

where $\mathbf{x}$ denotes the coarse-grained coordinate labeling a tissue patch and $a(\mathbf{x})$ describes the smoothed network coupling $a_{|i-j|}$. These generalized $\bar{g}(t)$ and $\sigma_{g}^{2}(t)$ are substituted in Equation (2.37) to obtain a coupled set of integro-differential kinetic equations for the voltage probability density $\rho^{(v)}(v, t ; \mathbf{x})$ and first conditional moment $\mu_{1}(v, t ; \mathbf{x})$. The same argument applies for the extension of the mean-field model, as well.

\section{Conclusions and discussion}

Kinetic theory provides an accurate and efficient population-based coarse-graining method for describing neuronal network dynamics [31,32, 98]. While the coarsegraining assumption underlying the kinetic theory is that of a large network, $N \gg 1$, so that a statistical description is meaningful, in practice, even for networks of size $N=\mathcal{O}(10)$ the kinetic theory captures large-fluctuation effects accurately and efficiently. As shown in Figures 2.2 and 2.3, the kinetic theory approximates full dynamical simulation results of the corresponding I\&F networks accurately both for 
stationary and time-dependent Poisson rates of the external drive, and both in the fluctuation- and mean-driven regimes. The computational savings afforded by simulating the kinetic equations instead of a large, I\&F, point-neuron model are significant, and arise from two sources: the first is the statistical nature of the kinetic equations, which eliminates the need for simulating large network ensembles, or equivalently, one network for long times, in order to collect statistically accurate results. The second is the dimension reduction achieved by our closure, which typically reduces computation time by 4 to 6 orders of magnitude within the accuracy of three significant digits $[31,32,98]$.

In addition to dramatically reducing the computational costs as compared to direct simulations of point-neuron networks, the kinetic theory and its further reductions also provide a powerful theoretical tool for the analysis of neuronal network dynamics. For example, as we have seen in Section 2.3.3, using the mean-field theory alone, one can already explain the presence of bistability in certain dynamical regimes of neuronal networks. As shown in Section 2.5.3, using the kinetic theory or its Fokker-Planck reduction, one can explain the gradual removal of this bistability when the amount of synaptic-input fluctuations increases [65]. As we have seen in Section 2.5.6, the corresponding bifurcation scenario of fluctuation-controlled criticality appears to be an important ingredient in the mechanism of neuronal orientation selectivity in the primary visual cortex [130].

Of course, the kinetic theory only describes the statistics of the neuronal voltages and membrane potentials, not the individual time-courses of these quantities for each network neuron, and the average neuronal firing rates, not an individual neuron's spike-times. When these more specific quantities are required, one must resort to full $I \& F$ network simulations. In cases when detailed dynamical information is only required for specific groups of neurons, a very promising but as yet largely unexplored modeling technique would consist of hybrids between coarse-grained and point-neuron models. One such hybrid between the kinetic theory and an I\&F point-neuron model was developed in [30].

An intermediate coarse-graining step would be a generalization of the kinetictheoretic approach presented here to more than one-point statistics, such as including the two-point correlation functions [68]. In fact, experimental measurements indicate that populations of neurons in various brain areas exhibit temporal correlations between firings $[7,38,64,67,77,104,106,109,115,146]$, which have been associated with stimulus encoding, discrimination, and behavior. The inability of the current kinetic theory to incorporate dynamical correlations among neuronal firings has prevented it from addressing a number of deep and interesting problems in realistic settings $[7,54,104]$, so that its generalization to multi-point statistics would be an important and timely effort.

Higher-order statistics of neuronal firings will also be strongly influenced by the network architecture, whose own statistical properties are not yet well understood. Experimental evidence points at the fact that neuronal networks can be sparse $[9,73-$ $76,118,133,134]$. Cortical networks appear to possess small-world attributes, with the numbers of synapses needed to connect a given pair of neurons close to those in similar random networks but with significantly more clustering [56, 57, 123, 124], scale-free topology [41], or perhaps both [59,121]. We have so far addressed firing-rate statistics of scale-free networks in the framework of the mean-field model deep into the mean-driven regime in order to decouple the effects of the network-connectivity fluctuations from those of the synaptic input-fluctuations. 
Many questions concerning coarse-grained dynamics of complex I\&F networks still remain. Some could potentially be answered using the mean-field model, such as whether network-connectivity fluctuations modify or remove the bistability of gain curves like the synaptic-input fluctuations do. Addressing small-world effects probably falls in this category as well. Formally extending our kinetic equations and their derivation via the maximum-entropy principle to the complex-network case is likely to be relatively straightforward; the extension of the computational algorithm of [98] may present a bigger challenge due to the numerical issues arising from the coupling in the dynamics of many populations of $k$-neurons. The main challenge seems to lie in the above-mentioned question of how to generalize the kinetic theory to the case of multi-point statistics and taking into account complex network topology at the same time.

A very general approach to this question from the viewpoint of the perturbation theory is presented in $[93,94]$. For weakly-coupled networks, successive approximations to the complete network statistics are found using an expansion in terms of the small coupling parameter. The $n$th approximation involves subnetworks of neurons coupled via $n$ intermediate synaptic connections. The work described in $[93,94]$ thus provides a potentially important probabilistic framework for investigating neuronal network dynamics.

Acknowledgment. The authors would like to thank R. Shapley and M. Shelley for fruitful discussions. D.C. was partly supported by NSF grants DMS-0211655, DMS-0506396, and DMS-1009575. L.T. was partly supported by NSF grant DMS0506257 and the National Basic Research Program of China (973 Program 2011-CB809105). M.S.S. was partly supported by the NSF grant DMS-0636358. A.V.R. was partly supported by NSF grant DMS-0914827. D.W.M. was partly supported by NSF grants DMS-0211655 and DMS-0506396. G.K. was partly supported by NSF grants DMS-0308943 and DMS-0506287.

Appendix A. Flowchart of equation interdependence. In this appendix, we display a flowchart representing the interdependence of the exact and approximate coarse-grained equations corresponding to the all-to-all coupled I\&F model, as discussed in the text of the paper. We denote the choice of description, limits, and assumptions leading to the specific equations by Roman numerals. The flowchart is displayed in Figure A.1.

Not shown in Figure A.1 is the progression from the advection-diffusion Equation (2.22) via the marginalization on the conductance to obtain the Fokker-Planck Equation (2.27), and further mean-driven limit and projection on the first conductance moment to obtain Equation (2.10b).

An analogous flowchart can be obtained for the heterogeneously coupled I\&F model (3.1), which includes nontrivial network topology, and replaces Equation (2.1) in the chart. The corresponding Boltzmann system of Equation (3.5) and advectiondiffusion Equation (3.9) replace Equations (2.3) and (2.22). The mean-field system (3.12) replaces the system (2.10), and its steady version (3.15) replaces the steady mean-field Equation (2.17a). Finally, the limiting, high-conductance system (3.16) replaces the equation for the linear gain-curve asymptote, Equation (2.20). Other complex-network analogs of the equations in the flowchart in Figure A.1 can be obtained using the techniques of this paper. 


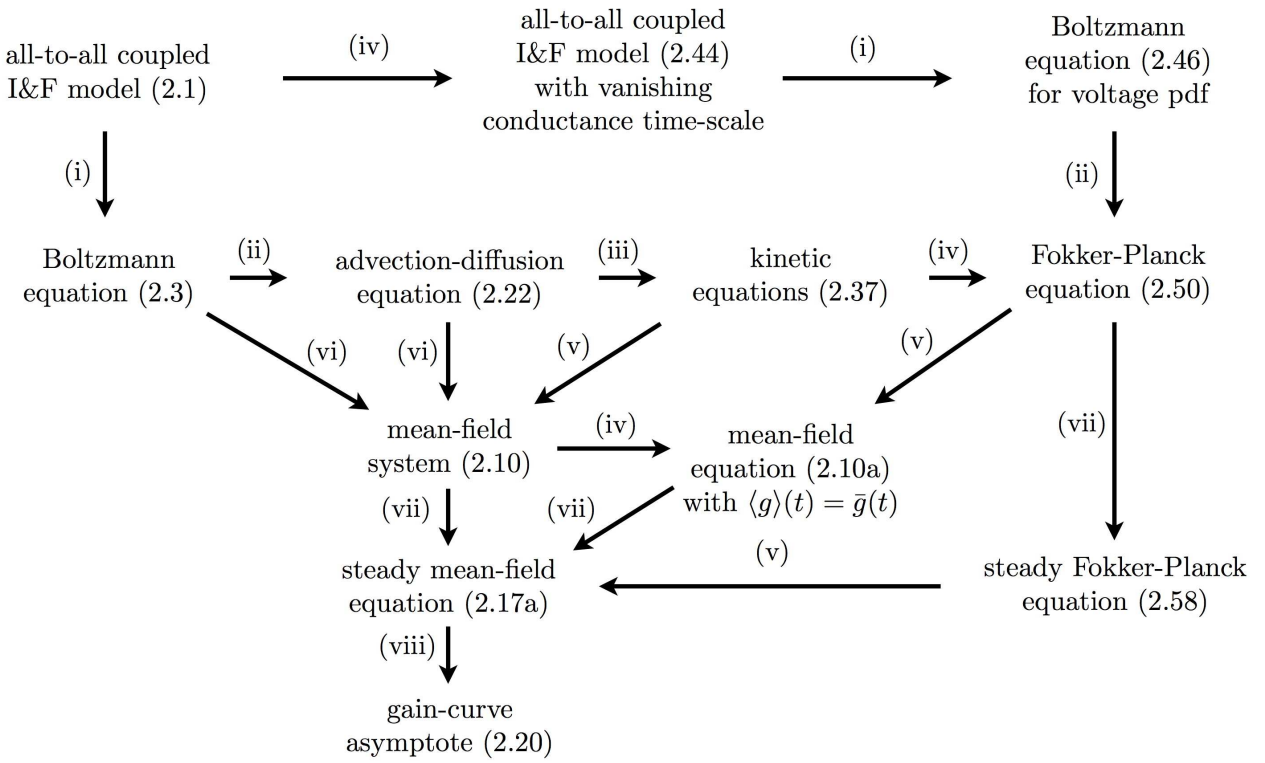

FIG. A.1. The flowchart for the equations, discussed in the text, corresponding to the all-to-all coupled IBFF model. The labels above the arrows represent: (i) statistical description; (ii) diffusion approximation; (iii) maximum-entropy closure; (iv) limit $\sigma \rightarrow 0$, (v) mean-driven limit $f \rightarrow 0, N, \nu \rightarrow$ $\infty$, with $f \nu=\mathcal{O}(1)$; (vi) same mean-driven limit as in $(v)$, assumption $\rho(v, g, t)=\rho^{(v)}(v, t) \rho^{(g)}(g, t)$, marginalization on the voltage and taking the first conductance moment; (vii) steady-state; (viii) high conductance.

\section{REFERENCES}

[1] L. Abbott and C. van Vreeswijk, Asynchronous states in networks of pulse-coupled oscillators, Phys. Rev. E, 48, 1483-1490, 1993.

[2] R.V. Abramov, M.J. Grote, and A.J. Majda, Information Theory and Stochastics for Multiscale Nonlinear Systems, CRM Monograph Series, American Mathematical Society, Centre de Recherches Mathématiques, Université de Montréal, 25, 2005.

[3] H.J. Alitto and W.M. Usrey, Influence of contrast on orientation and temporal frequency tuning in ferret primary visual cortex, J. Neurophysiol., 91, 2797-2808, 2004.

[4] D.J. Amit and N. Brunel, Model of global spontaneous activity and local structured activity during delay periods in the cerebral cortex, Cereb. Cortex, 7, 237-252, 1997.

[5] J. Anderson, I. Lampl, D. Gillespie, and D. Ferster, The contribution of noise to contrast invariance of orientation tuning in cat visual cortex, Science, 290, 1968-1972, 2000.

[6] J. Anderson, I. Lampl, I. Reichova, M. Carandini, and D. Ferster, Stimulus dependence of twostate fluctuations of membrane potential in cat visual cortex, Nat. Neurosci., 3, 617-621, 2000.

[7] B.B. Averbeck, P.E. Latham, and A. Pouget, Neural correlations, population coding and computation, Nat. Rev. Neurosci., 7, 358-366, 2006.

[8] G. Barna, T. Grobler, and P. Erdi, Statistical model of the hippocampal ca3 region, ii. The population framework: Model of rhythmic activity in ca3 slice, Biol. Cybern., 79, 309-321, 1998

[9] P. Bartho, H. Hirase, L. Monconduit, M. Zugaro, K.D. Harris, and G. Buzsaki, Characterization of neocortical principal cells and interneurons by network interactions and extracellular features, J. Neurophysiol., 92, 600-608, 2004.

[10] R. Ben-Yishai, R. Bar-Or, and H. Sompolinsky, Theory of orientation tuning in the visual cortex, Proc. Natl. Acad. Sci. USA, 92, 3844-3848, 1995.

[11] G. Blasdel, Differential imaging of ocular dominance and orientation selectivity in monkey striate cortex, J. Neurosci., 12, 3115-3138, 1992. 
[12] G. Blasdel, Orientation selectivity, preference, and continuity in the monkey striate cortex, J. Neurosci., 12, 3139-3161, 1992.

[13] S. Boccaletti, V. Latora, Y. Moreno, M. Chavez, and D.U. Hwang, Complex networks: Structure and dynamics, Physics Reports, 424, 175-308, 2006.

[14] L. Boltzmann, Studien uber das gleichgewicht der lebendigen kraft zwischen bewegten $m$ ateriellen punkten, Wien. Ber., 58, 1868; reprinted in Boltzmann Abhandlungen, Barth, Leipzig, 1, 49-517, 1909.

[15] L. Boltzmann, Weitere studien uber das warmegleichgewicht unter gasmolekulen, Wien. Ber., 66, 1872, reprinted in Boltzmann Abhandlungen, Barth, Leipzig, 1, 275-316, 1909.

[16] T. Bonhoeffer and A. Grinvald, Iso-orientation domains in cat visual cortex are arranged in pinwheel like patterns, Nature, 353, 429-431, 1991.

[17] P. Bressloff, J. Cowan, M. Golubitsky, P. Thomas, and M. Wiener, Geometric visual hallucinations, euclidean symmetry and the functional architecture of striate cortex, Phil. Trans. R. Soc. Lond. B, 356, 299-330, 2001.

[18] P. Bressloff, J. Cowan, M. Golubitsky, P. Thomas, and M. Wiener, What geometric visual hallucinations tell us about the visual cortex, Neural Computation, 14, 473-491, 2002.

[19] P.C. Bressloff, Stochastic neural field theory and the system-size expansion, SIAM J. Appl. Math., 70, 1488-1521, 2009.

[20] P.C. Bressloff, Metastable states and quasicycles in a stochastic wilson-cowan model of neuronal population dynamics, Phys. Rev. E, 82, 051903, 2010.

[21] P.C. Bressloff and Y.M. Lai, Stochastic synchronization of neuronal populations with intrinsic and extrinsic noise, J. Math. Neurosci., 1, 2011.

[22] R. Brette, M. Rudolph, T. Carnevale, M. Hines, D. Beeman, J.M. Bower, M. Diesmann, A. Morrison, P.H. Goodman, F.C. Harris Jr, M. Zirpe, T. Natschlager, D. Pecevski, B. Ermentrout, M. Djurfeldt, A. Lansner, O. Rochel, T. Vieville, E. Muller, A.P. Davison, S.E. Boustani, and A. Destexhe, Simulation of networks of spiking neurons: A review of tools and strategies, J. Comput. Neurosci., 23, 349-398, 2007.

[23] N. Brunel, Dynamics of networks of randomly connected excitatory and inhibitory spiking neurons, J. Physiol. Paris, 94, 445-463, 2000.

[24] N. Brunel and V. Hakim, Fast global oscillations in networks of integrate-and-fire neurons with low firing rates, Neural Comput., 11, 1621-1671, 1999.

[25] N. Brunel and S. Sergi, Firing frequency of leaky integrate-and-fire neurons with syncaptic current dynamics, J. Theoretical Biology, 195, 87-95, 1998.

[26] M.A. Buice and J.D. Cowan, Field-theoretic approach to fluctuation effects in neural networks, Phys. Rev. E, 75, 051919, 2007.

[27] M.A. Buice and J.D. Cowan, Statistical mechanics of the neocortex, Prog. Biophys. Mol. Biol., 99, 53-86, 2009.

[28] M.A. Buice, J.D. Cowan, and C.C. Chow, Systematic fluctuation expansion for neural network activity equations, Neural Comput., 22, 377-426, 2010.

[29] D. Cai, A. Rangan, and D. McLaughlin, Architectural and synaptic mechanisms underlying coherent spontaneous activity in V1, Proc. Natl. Acad. Sci. USA, 102, 5868-5873, 2005.

[30] D. Cai, L. Tao, and D.W. McLaughlin, An embedded network approach for scale-up of fluctuation-driven systems with preservation of spike information, Proc. Natl. Acad. Sci. USA, 101, 14288-14293, 2004

[31] D. Cai, L. Tao, A.V. Rangan, and D.W. McLaughlin, Kinetic theory for neuronal network dynamics, Commun. Math. Sci., 4, 97-127, 2006.

[32] D. Cai, L. Tao, M. Shelley, and D. McLaughlin, An effective representation of fluctuationdriven neuronal networks with application to simple 83 complex cells in visual cortex, Pro. Natl. Acad. Sci. USA, 101, 7757-7762, 2004

[33] N. Carnevale and M. Hines, The NEURON Book, Cambridge University Press, Cambridge, 2006.

[34] A. Casti, A. Omurtag, A. Sornborger, E. Kaplan, B. Knight, J. Victor, and L. Sirovich, A population study of integrate-and-fire-or-burst neurons, Neural Comput., 14, 957-986, 2002.

[35] M. Catanzaro, M. Boguna, and R. Pastor-Satorras, Generation of uncorrelated random scalefree networks, Phys. Rev. E, 71, 027103, 2005.

[36] T. Chawanya, A. Aoyagi, T. Nishikawa, K. Okuda, and Y. Kuramoto, A model for feature linking via collective oscillations in the primary visual cortex, Biol. Cybern., 68, 483-490, 1993.

[37] E. Cinlar, Superposition of point processes, in Stochastic Point Processes: Statistical Analysis, Theory, and Applications, P. Lewis (ed.), Wiley, New York, NY, 549-606, 1972.

[38] Y. Dan, J. Alonso, W. Usrey, and R. Reid, Coding of visual information by precisely correlated 
spikes in the lateral geniculate nucleus, Nat. Neurosci., 1, 501-507, 1998.

[39] G. DeAngelis, R. Ghose, I. Ohzawa, and R. Freeman, Functional micro-organization of primary visual cortex: Receptive field analysis of nearby neurons, J. Neurosci., 19, 40464064, 1999.

[40] S.N. Dorogovtsev and J.F.F. Mendes, Evolution of networks, Adv. Phys., 51, 1079-1187, 2002.

[41] V.M. Eguíluz, D.R. Chialvo, G.A. Cecchi, M. Baliki, and A.V. Apkarian, Scale-free brain functional networks, Phys. Rev. Lett., 94, 018102, 2005.

[42] O. Erdös and A. Rényi, On random graphs. i, Publicationes Mathematicae, 6, 290-297, 1959.

[43] O. Erdös and A. Rényi, The evolution of random graphs, Magyar Tud. Akad. Mat. Kutató Int. Közl., 5, 1960.

[44] R. Everson, A. Prashanth, M. Gabbay, B. Knight, L. Sirovich, and E. Kaplan, Representation of spatial frequency and orientation in the visual cortex, Proc. Natl. Acad. Sci. USA, 95, 8334-8338, 1998.

[45] D.A. Fair, A.L. Cohen, J.D. Power, N.U.F. Dosenbach, J.A. Church, F.M. Miezin, B.L. Schlaggar, and S.E. Petersen, Functional brain networks develop from a "local to distributed" organization, PLoS Comput. Biol, 5,1000381, 2009.

[46] D. Ferster and K.D. Miller, Neural mechanisms of orientation selectivity in the visual cortex, Ann. Rev. Neurosci., 23, 441-471, 2000.

[47] I.M. Finn, N.J. Priebe, and D. Ferster, The emergence of contrast-invariant orientation tuning in simple cells of cat visual cortex, Neuron, 54, 137-152, 2007.

[48] N. Fourcaud and N. Brunel, Dynamics of the firing probability of noisy integrate-and-fire neurons, Neural Comput., 14, 2057-2110, 2002.

[49] C.W. Gardiner, Handbook of Stochastic Methods, Springer, third ed., 2004.

[50] W. Gerstner, Population dynamics of spiking neurons: Fast transients, asynchronous states, and locking, Neural Comput., 12, 43-89, 2000.

[51] E.N. Gilbert, Random graphs, Ann. Math. Statist., 30, 1141-1144, 1959.

[52] M. Guye, F. Bartolomei, and J.P. Ranjeva, Imaging structural and functional connectivity: Towards a unified definition of human brain organization? Curr. Opin. Neurol., 21, 393440, 2008.

[53] D. Hansel, G. Mato, and C. Meunier, Synchrony in excitatory neural networks, Neural Comput., 7, 307-337, 1995 .

[54] K. Harris, Neural signatures of cell assembly organization, Natl. Rev. Neurosci, 6, 399-407, 2005.

[55] E. Haskell, D. Nykamp, and D. Tranchina, Population density methods for large-scale modeling of neuronal networks with realistic synaptic kinetics: Cutting the dimension down to size, Network: Compt. Neural. Syst., 12, 141-174, 2001.

[56] C.C. Hilgetag, G.A. Burns, M.A. O'Neill, J.W. Scannell, and M.P. Young, Anatomical connectivity defines the organization of clusters of cortical areas in the macaque monkey and the cat, Philos. Trans. R. Soc. Lond. B Biol. Sci., 355, 91-110, 2000.

[57] C. Honey, R. Kotter, M. Breakspear, and O. Sporns, Network structure of cerebral cortex shapes functional connectivity on multiple time scales, Proc. Natl. Acad. Sci. USA, 104, 10240-10245, 2007.

[58] A.A. Ioannides, Dynamic functional connectivity, Curr. Opin. Neurobiol., 17, 161-170, 2007.

[59] M. Kaiser, R. Martin, P. Andras, and M. Young, Simulation of robustness against lesions of cortical networks, Euro. J. Neurosci., 25, 3185-3192, 2007.

[60] Z.F. Kisvarday, P. Buzas, and U.T. Eysel, Calculating direction maps from intrinsic signals revealed by optical imaging, Cereb Cortex, 11, 636-647, 2001.

[61] K. Klemm and V.M. Eguíluz, Growing scale-free networks with small-world behavior, Phys. Rev. E, 65, 057102, 2002.

[62] B. Knight, Dynamics of encoding in a populaton neurons, J. Gen. Physiol., 59, 734-766, 1972.

[63] C. Koch, Biophysics of Computation, Oxford University Press, Oxford, 1999.

[64] A. Kohn and M. A. Smith, Stimulus dependence of neuronal correlation in primary visual cortex of the macaque, J. Neurosci., 25, 3661-3673, 2005.

[65] G. Kovačič, L. Tao, A.V. Rangan, and D. Cai, Fokker-planck description of conductance-based integrate-and-fire neuronal networks, Phys. Rev. E, 80, 021904, 2009.

[66] P.L. Krapivsky and S. Redner, Organization of growing random networks, Phys. Rev. E, 63, 066123, 2001.

[67] T. Lauritzen, A. Krukowski, and K. Miller, Local correlation-based circuitry can account for responses to multi-grating stimuli in a model of cat v1, J. Neurophysiol., 86, 1803-1815, 2001.

[68] C.Y. Liu and D.Q. Nykamp, A kinetic theory approach to capturing interneuronal correlation: The feed-forward case, J. Comput. Neurosci., 26, 339-368, 2009. 
[69] C. Ly and D. Tranchina, Critical analysis of dimension reduction by a moment closure method in a population density approach to neural network modeling, Neural Comput., 19, 20322092, 2007.

[70] Z.F. Mainen and T.J. Sejnowski, Reliability of spike timing in neocortical neurons, Science, 268, 1503-1506, 1995.

[71] A.J. Majda and X. Wang, Nonlinear Dynamics and Statistical Theories for Basic Geophysical Flows, Cambridge University Press, Cambridge, New York, 2006.

[72] P. Maldonado, I. Godecke, C. Gray, and T. Bonhoeffer, Orientation selectivity in pinwheel centers in cat striate cortex, Science, 276, 1551-1555, 1997.

[73] H. Markram, A network of tufted layer 5 pyramidal neurons, Cereb. Cortex, 7, 523-533, 1997.

[74] H. Markram, J. Lubke, M. Frotscher, A. Roth, and B. Sakmann, Physiology and anatomy of synaptic connections between thick tufted pyramidal neurones in the developing rat neocortex, J. Physiol., 500, 409-440, 1997.

[75] H. Markram, J. Lubke, M. Frotscher, and B. Sakmann, Regulation of synaptic efficacy by coincidence of postsynaptic aps and epsps, Science, 275, 213-215, 1997.

[76] H. Markram and M. Tsodyks, Redistribution of synaptic efficacy between neocortical pyramidal neurons, Nature, 382, 807-810, 1996.

[77] D.N. Mastronarde, Correlated firing of cat retinal ganglion cells. I. Spontaneously active inputs to $x$ - and $y$-cells, J. Neurophysiol., 49, 303-324, 1983.

[78] D. McLaughlin, R. Shapley, M. Shelley, and J. Wielaard, A neuronal network model of macaque primary visual cortex (V1): Orientation selectivity and dynamics in the input layer 4C $\alpha$, Proc. Natl. Acad. Sci. USA, 97, 8087-8092, 2000.

[79] R.E. Mirollo and S.H. Strogatz, Synchronization of pulse-coupled biological oscillators, SIAM J. Appl. Math., 50, 1645-1662, 1990

[80] K.A. Newhall, G. Kovačič, P.R. Kramer, and D. Cai, Cascade-induced synchrony in stochastically-driven neuronal networks, Phys. Rev. E, 82, 041903, 2010.

[81] K.A. Newhall, G. Kovačič, P.R. Kramer, D. Zhou, A.V. Rangan, and D. Cai, Dynamics of current-based, Poisson driven, integrate-and-fire neuronal networks, Commun. Math. Sci., 8, 541-600, 2010.

[82] M. Newman, The structure and function of complex networks, SIAM Rev., 45, 167-256, 2003.

[83] M.E. Newman, S.H. Strogatz, and D.J. Watts, Random graphs with arbitrary degree distributions and their applications, Phys. Rev. E, 64, 026118, 2001.

[84] M.E.J. Newman, Assortative mixing in networks, Phys. Rev. Lett., 89, 208701, 2002.

[85] M.E.J. Newman, D.J. Watts, and S.H. Strogatz, Random graph models of social networks, Proc. Natl. Acad. Sci. USA, 99, 2566-2572, 2002.

[86] L.G. Nowak, M.V. Sanchez-Vives, and D.A. McCormick, Influence of low and high frequency inputs on spike timing in visual cortical neurons, Cereb. Cortex, 7, 487-501, 1997.

[87] D. Nykamp and D. Tranchina, A population density method that facilitates large-scale modeling of neural networks: Analysis and application to orientation tuning, J. Comput. Neurosci., 8, 19-50, 2000.

[88] D. Nykamp and D. Tranchina, A population density method that facilitates large-scale modeling of neural networks: Extension to slow inhibitory synapses, Neural Comput., 13, 511-546, 2001.

[89] A. Omurtag, E. Kaplan, B. Knight, and L. Sirovich, A population approach to cortical dynamics with an application to orientation tuning, Network, 11, 247-260, 2000.

[90] A. Omurtag, B. Knight, and L. Sirovich, On the simulation of large populations of neurons, J. Comput. Neurosci., 8, 51-63, 2000.

[91] J. Pham, K. Pakdaman, J. Champagnat, and J. Vibert, Activity in sparsely connected excitatory neural networks: Effect of connectivity neural networks, Neural Networks, 11, 415-434, 1998.

[92] N. Ramnani, T.E.J. Behrens, W. Penny, and P.M. Matthews, New approaches for exploring anatomical and functional connectivity in the human brain, Biol. Psychiatry, 56, 613-619, 2004

[93] A.V. Rangan, Diagrammatic expansion of pulse-coupled network dynamics, Phys. Rev. Lett., 102, 158101, 2009.

[94] A.V. Rangan, Diagrammatic expansion of pulse-coupled network dynamics in terms of subnetworks, Phys. Rev. E, 80, 036101, 2009.

[95] A.V. Rangan and D. Cai, Maximum-entropy closures for kinetic theories of neuronal network dynamics, Phys. Rev. Lett., 96, 178101, 2006.

[96] A.V. Rangan and D. Cai, Fast numerical methods for simulating large-scale integrate-and-fire neuronal networks, J. Comput. Neurosci., 22, 81-100, 2007.

[97] A.V. Rangan, D. Cai, and D.W. McLaughlin, Modeling the spatiotemporal cortical activity 
associated with the line-motion illusion in primary visual cortex, Proc. Natl. Acad. Sci. USA, 102, 18793-18800, 2005.

[98] A.V. Rangan, D. Cai, and L. Tao, Numerical methods for solving moment equations in kinetic theory of neuronal network dynamics, J. Comput. Phys., 221, 781-798, 2007.

[99] A.V. Rangan, G. Kovačič, and D. Cai, Kinetic theory for neuronal networks with fast and slow excitatory conductances driven by the same spike train, Phys. Rev. E, 77, 041915, 2008 .

[100] A.V. Rangan, L. Tao, G. Kovačič, and D. Cai, Large-scale computational modeling of the primary visual cortex, in Coherent Behavior in Neuronal Networks, K. Josić, M. Matias, R. Romo, and J. Rubin (eds.), Springer Series in Computational Neuroscience, SpringerVerlag, 3, 2009.

[101] A.V. Rangan, L. Tao, G. Kovačič, and D. Cai, Multi-scale modeling of the primary visual cortex, IEEE Engineering in Medicine and Biology Magazine, 28, 19-24, 2009.

[102] J.C. Reijneveld, S.C. Ponten, H.W. Berendse, and C.J. Stam, The application of graph theoretical analysis to complex networks in the brain, Clin. Neurophysiol., 118, 2317-2331, 2007.

[103] B. Roerig and J.P. Kao, Organization of intracortical circuits in relation to direction preference maps in ferret visual cortex, J. Neurosci., 19, RC44, 1999.

[104] E. Salinas and T.J. Sejnowski, Correlated neuronal activity and the flow of neural information, Nat. Rev. Neurosci., 2, 539-550, 2001.

[105] S. Sato, On the moments of the firing interval of the diffusion approximated model neuron, Math. Bio., 39, 53-70, 1978.

[106] E. Schneidman, M.J.N. Berry, R. Segev, and W. Bialek, Weak pairwise correlations imply strongly correlated network states in a neural population, Nature, 440, 1007-1012, 2006.

[107] G. Sclar and R. Freeman, Orientation selectivity in the cat's striate cortex is invariant with stimulus contrast, Exp. Brain Research, 46, 457-461, 1982.

[108] M. Shadlen and W. Newsome, Noise, neural codes and cortical organization, Curr. Opin. Neurobiol., 4, 569-579, 1994.

[109] M. Shadlen and W. Newsome, The variable discharge of cortical neurons: Implications for connectivity, computation and information coding, J. Neurosci., 18, 3870-3896, 1998.

[110] R. Shapley, M. Hawken, and D.L. Ringach, Dynamics of orientation selectivity in the primary visual cortex and the importance of cortical inhibition, Neuron, 38, 689-699, 2003.

[111] R. Shapley, M. Hawken, and D. Xing, The dynamics of visual responses in the primary visual cortex, Prog. Brain Res., 165, 21-32, 2007.

[112] M. Shelley and D. McLaughlin, Coarse-grained reduction and analysis of a network model of cortical response. I. Drifting grating stimuli, J. Comput. Neurosci., 12, 97-122, 2002.

[113] M.S. Shkarayev, G. Kovačič, and D. Cai, Topological effects on dynamics in complex pulsecoupled networks of integrate-and-fire type, submmitted to Phys. Rev. E, 2011.

[114] M.S. Shkarayev, G. Kovačič, A.V. Rangan, and D. Cai, Architectural and functional connectivity in scale-free integrate-and-fire networks, Europhys. Lett., 88, 50001, 2009.

[115] J. Shlens, G.D. Field, J.L. Gauthier, M.I. Grivich, D. Petrusca, A. Sher, A.M. Litke, and E.J. Chichilnisky, The structure of multi-neuron firing patterns in primate retina, J. Neurosci., 26, 8254-8266, 2006.

[116] A. Shmuel and A. Grinvald, Functional organization for direction of motion and its relationship to orientation maps in cat area 18, J. Neurosci., 16, 6945-6964, 1996.

[117] G. Silberberg, M. Bethge, H. Markram, K. Pawelzik, and M. Tsodyks, Dynamics of Population Rate Codes in Ensembles of Neocortical Neurons, J. Neurophysiol., 91, 704-709, 2004.

[118] P.J. Sjostrom, G.G. Turrigiano, and S.B. Nelson, Rate, timing, and cooperativity jointly determine cortical synaptic plasticity, Neuron., 32, 1149-1164, 2001.

[119] B.C. Skottun, A. Bradley, G. Sclar, I. Ohzawa, and R.D. Freeman, The effects of contrast on visual orientation and spatial frequency discrimination: A comparison of single cells and behavior, J. Neurophysiol, 57, 773-786, 1987.

[120] D. Somers, S. Nelson, and M. Sur, An emergent model of orientation selectivity in cat visual cortical simple cells, J. Neurosci., 15, 5448-5465, 1995.

[121] O. Sporns, D. Chialvo, M. Kaiser, and C. Hilgetag, Organization, development and function of complex brain networks, Trends Cogn. Sci., 8, 418-425, 2004.

[122] O. Sporns, D.R. Chialvo, M. Kaiser, and C.C. Hilgetag, Organization, development and function of complex brain networks, Trends Cogn. Sci., 8, 418-425, 2004.

[123] O. Sporns, G. Tononi, and G.M. Edelman, Theoretical neuroanatomy: Relating anatomical and functional connectivity in graphs and cortical connection matrices, Cereb. Cortex, $10,127-141,2000$.

[124] O. Sporns and J.D. Zwi, The small world of the cerebral cortex, Neuroinformatics, 2, 145-162, 
2004

[125] C.J. Stam, Characterization of anatomical and functional connectivity in the brain: A complex networks perspective, Int. J. Psychophysiol., 77, 186-194, 2010.

[126] C.J. Stam and J.C. Reijneveld, Graph theoretical analysis of complex networks in the brain, Nonlinear Biomed. Phys., 1, 3, 2007.

[127] C.F. Stevens and A.M. Zador, Input synchrony and the irregular firing of cortical neurons, Nat. Neurosci., 1, 210-217, 1998.

[128] S. Strogatz, Exploring complex networks, Nature, 410, 268-276, 2001.

[129] N.V. Swindale, A. Grinvald, and A. Shmuel, The spatial pattern of response magnitude and selectivity for orientation and direction in cat visual cortex, Cereb Cortex, 13, 225-238, 2003.

[130] L. Tao, D. Cai, D. McLaughlin, M. Shelley, and R. Shapley, Orientation selectivity in visual cortex by fluctuation-controlled criticality, Proc. Natl. Acad. Sci. USA, 103, 12911-12916, 2006

[131] L. Tao, M. Shelley, D. McLaughlin, and R. Shapley, An egalitarian network model for the emergence of simple and complex cells in visual cortex, Proc. Natl. Acad. Sci. USA, 101, 366-371, 2004

[132] N.M. Temme, Uniform asymptotics for the incomplete gamma functions starting from negative values of the parameters, Meth. Appl. Anal., 3, 335-344, 1996.

[133] A.M. Thomson and A.P. Bannister, Interlaminar connections in the neocortex, Cereb. Cortex, $13,5-14,2003$.

[134] A.M. Thomson, D.C. West, Y. Wang, and A.P. Bannister, Synaptic connections and small circuits involving excitatory and inhibitory neurones in layers 2 to 5 of adult rat and cat neocortex: Triple intracellular recordings and biocytin-labelling in vitro, Cereb. Cortex, 12, 936-953, 2002.

[135] A. Treves, Mean field analysis of neuronal spike dynamics, Network, 4, 259-284, 1993.

[136] T. Troyer, A. Krukowski, N. Priebe, and K. Miller, Contrast invariant orientation tuning in cat visual cortex with feedforward tuning and correlation based intracortical connectivity, J. Neurosci., 18, 5908-5927, 1998.

[137] M.P. van den Heuvel, C.J. Stam, M. Boersma, and H.E. Hulshoff Pol, Small-world and scalefree organization of voxel-based resting-state functional connectivity in the human brain, Neuroimage, 43, 528-539, 2008.

[138] W. Vanduffel, R. Tootell, A. Schoups, and G. Orban, The organization of orientation selectivity throughout macaque visual cortex, Cereb. Cortex, 12, 647-662, 2002.

[139] M. Volgushev, J. Pernberg, and U.T. Eysel, A novel mechanism of response selectivity of neurons in cat visual cortex, J. Physiol., 540, 307-320, 2002.

[140] M. Volgushev, J. Pernberg, and U.T. Eysel, Gamma-frequency fluctuations of the membrane potential and response selectivity in visual cortical neurons, Eur. J. Neurosci., 17, 17681776,2003

[141] D.J. Watts and S.H. Strogatz, Collective dynamics of 'small-world' networks, Nature, 393 , 440-442, 1998.

[142] M. Weliky, W.H. Bosking, and D. Fitzpatrick, A systematic map of direction preference in primary visual cortex, Nature, 379, 725-728, 1996.

[143] W. Wilbur and J. Rinzel, A theoretical basis for large coefficient of variation and bimodality in neuronal interspike interval distributions, J. Theor. Biol., 105, 345-368, 1983.

[144] H. Wilson and J. Cowan, A mathematical theory of the functional dynamics of cortical and thalamic nervous tissue, Kybernetik, 13, 55-80, 1973.

[145] H.R. Wilson and J.D. Cowan, Excitatory and inhibitory interactions in localized populations of model neurons, Biophys. J., 12, 1-24, 1972.

[146] E. Zohary, M. Shadlen, and W. Newsome, Correlated neuronal discharge rate and its implication for psychophysical performance, Nature, 370, 140-143, 1994. 